\title{
Interférences
}

Ars scribendi

$6 \mid 2012$

La question de la littérarité

\section{Quelques réflexions sur la notion de littérarité à partir de l'édition numérique de commentateurs anciens}

Bruno Bureau

\section{CpenEdition}

Journals

Édition électronique

URL : http://journals.openedition.org/interferences/186

DOI : 10.4000/interferences. 186

ISSN : $1777-5485$

Éditeur

HiSoMA - Histoire et sources des Mondes antiques

Édition imprimée

Date de publication : 1 janvier 2012

ISSN : 1777-5485

Référence électronique

Bruno Bureau, «Quelques réflexions sur la notion de littérarité à partir de l'édition numérique de commentateurs anciens », Interférences [En ligne], 6 | 2012, mis en ligne le 11 décembre 2014, consulté le 15 septembre 2020. URL : http://journals.openedition.org/interferences/186 ; DOI : https:// doi.org/10.4000/interferences. 186

Ce document a été généré automatiquement le 15 septembre 2020.

Tous droits réservés 


\title{
Quelques réflexions sur la notion de littérarité à partir de l'édition numérique de commentateurs anciens
}

\author{
Bruno Bureau
}

\section{Introduction}

1 En lisant le programme du colloque de Nanterre sur la notion de littérarité dans l'Antiquité ${ }^{1}$, nous nous sommes arrêté sur cette phrase qui nous parait décrire parfaitement la zone de malentendu actuelle autour des objets numériques et de leur rapport à la " philologie » dite classique : « dans le moment actuel de basculement et de crise culturelle, la disparition annoncée du livre de papier au profit des multiples formes de l'électronique n'apparaît pas comme le passage d'un support à un autre, et comme la simple évolution d'un vecteur, mais comme la transformation radicale de la notion même de texte ». Dans cette approche, on reconnait aisément la mise en œuvre d'une opposition entre le livre - que beaucoup voient comme forcément appelé à disparaître ou à muter sous la forme de «tablettes» et autres objets alliant fonctionnalités de lecture "simple» et technologies numériques - et de nouvelles formes de communication que l'on soupçonne plus ou moins de vouloir sa fin. De tels débats ne sont pas nouveaux ${ }^{2}$ mais, dans un colloque sur la littérarité, leur réapparition nous paraît typique du lien que nous établissons tous, volontairement ou non, entre livre et littérarité, comme si l'un ne pouvait aller sans l'autre.

2 Notre propos sera ici d'intervenir en tant qu'éditeur numérique peut-être aussi un peu philologue et de nous poser la question suivante : si nous avons peur d'une absorption de la littérarité par le numérique, n'est-ce pas simplement que nous avons des idées préconçues de la littérarité qui font du numérique un danger potentiel et non « un ami qui nous veut du bien»? Cela suppose évidemment que nous savons le faire servir à 
notre enquête, mais aussi nous laisser interroger par les possibilités qu'il offre et relire l'épistémologie de notre discipline à la lumière de ce qu'il éclaire ${ }^{3}$.

3 Il est évident que le numérique entraîne, qu'on le veuille ou non, une mutation du mode de lecture, le nier serait mentir. Mais cette mutation, à notre sens, si elle questionne la notion de littérarité, ne la remet pas en cause, bien au contraire. Il nous semble qu'elle nous oblige à la chercher là où elle est, et non dans la facilité du livre, qui nous la livre en quelque sorte préconditionnée. Mais par qui, par quoi ou pour quoi ? Ce que nous redoutons finalement, c'est sans doute la modification de notre mode de lecture, la perte de la rassurante linéarité du livre, mais est-ce bien essentiel à notre présent objet? L'affirmer serait lier la littérarité au livre lui-même, ce qui n'est pas en soi impensable, mais demande quand même à être questionné pour nous assurer que nous ne passons pas, s'agissant de l'Antiquité où la pratique du livre est très différente de la nôtre, à côté d'enjeux majeurs.

Plus largement, ce qui est essentiel dans cette réflexion sur la littérarité face aux nouveaux modes d'édition, c'est la notion même de texte, notion que nous lions ordinairement à sa représentation livresque, mais qui est sans doute, y compris pour les Latins eux-mêmes, bien autre chose. Dire cela, autrement dit affirmer que ce que nous appelons texte livresque n'est pas le texte des Anciens et n'est plus celui des concepteurs d'objets numériques, c'est déjà établir la possibilité d'un dialogue entre la civilisation ancienne et les outils les plus modernes, non pas en éliminant le livre, mais en le comprenant comme ce qu'il est: une forme particulière de discours sur la littérarité ${ }^{4}$.

On peut alors poser le problème autrement et envisager le rapport du texte (indépendamment de son support de diffusion) à la littérarité.

Dans cette enquête, les commentaires antiques dont nous nous occupons constituent un excellent terrain d'expérience, parce qu'ils ne cessent d'interroger précisément cette notion de littérarité dans ses multiples dimensions.

7 En tant que discours critique sur des textes, ils offrent des lectures antiques de ce qui constitue la littérarité, qu'ils s'efforcent de quantifier, d'évaluer (bene, recte ${ }^{5}$ ), de qualifier (bona figura ${ }^{6} .$. ), de replacer dans son contexte de production (ueteres sic, nos aliter ${ }^{7}$ ). Pour les grammairiens qui les composent, comme sans doute pour une grande partie de la tradition romaine, ce que nous appelons littérarité peut se définir ainsi : la conformité d'un discours aux règles qui sont supposées le régir, et le fait de porter ces règles à un point où le discours devient d'une certaine manière un modèle digne d'être imité ou reproduit ${ }^{8}$. Sur ce point, les textes de commentaire sont essentiels à notre sujet parce qu'ils nous transmettent un discours romain sur la littérarité et ses critères.

8 Pourtant, si l'on consulte les histoires de la littérature latine pour le grand public, on n'y trouve pas ces textes qui ne sont pas jugés assez littéraires pour avoir place aux côtés d'Ausone, Claudien, Prudence ou Ammien. Ils rejoignent dans les notes ou les petits paragraphes de résumé d'autres ostracisés de la littérarité comme Végèce, Pélagonius, Palladius et autres «auteurs techniques", pour ne rien dire des grammairiens. Et là se trouve un premier paradoxe: il ne viendrait à l'idée d'aucun auteur d'une littérature tardive de ne pas citer parmi les œuvres d'Augustin les traités sur saint Jean ou les Enarrationes in Psalmos qui pourtant empruntent l'essentiel de leur technique littéraire au commentaire grammatical. Faut-il donc être chrétien pour écrire un commentaire qui accède à la littérarité ? On verra que la question mérite d'être posée, mais pour l'instant tenons-nous en à noter que le genre du commentaire, 
toutes confessions religieuses et toutes époques confondues, est un genre à la littérarité problématique donc intéressante, car il interroge finalement ce que nous entendons par « littérarité », et ce qui constitue un texte en texte littéraire.

\section{De l'oral à l'écrit, un modèle oratoire de littérarité}

\section{Pensée du discours et pensée du texte}

En fait, la première étape est sans doute de se mettre d'accord sur ce que nous entendons par texte, et ce qu'entendent par là les Latins. Sans cela, toute tentative d'édition ne saurait garantir la conformité de sa méthode à son objet même, ce qui, épistémologiquement, n'est pas sans conséquence. En effet, ne pas prendre en compte le statut même du texte que l'on édite aboutit très rapidement à un non-sens. Qui songerait à choisir dans l'édition de Virgile une variante manuscrite métriquement impossible?

Pour dire très clairement vers quoi tend ici mon propos, il me semble que ce n'est ni la forme textuelle qui fait la littérarité, ni la littérarité qui conditionne la forme textuelle. Autrement dit, il me semble qu'il faut dissocier littérarité et textualité ${ }^{9}$. C'est un peu un truisme aujourd'hui, mais c'est un truisme fondamental pour aborder la littérarité latine. On notera d'abord que le mot textus n'en vient que tardivement à désigner le texte et que, dans les écrits de la période classique, un texte se définit d'abord par des éléments génériques : epistula, carmen, narratio, oratio, comoedia, commentarii, etc. C'est donc primitivement l'usage du langage conformément à un code générique, et non la forme même que reçoit le résultat, texte ou discours, qui définit la littérarité. Les Latins ont donc conçu la littérarité indépendamment de la textualité, un ouvrage littéraire pouvant très bien ne pas être ce que nous appelons aujourd'hui un texte, c'est-à-dire un discours figé sur un support écrit.

11 Pour dire les choses clairement, la littérarité latine ne part pas du texte, elle y aboutit. Ainsi dit Cicéron (Tusc. 4, 55) ${ }^{10}$ :

Oratorem vero irasci minime decet, simulare non $<$ de>decet. An tibi irasci tum videmur, cum quid in causis acrius et vehementius dicimus? Quid? cum iam rebus transactis et praeteritis orationes scribimus, num irati scribimus?

'Ecquis hoc animadvertit? vincite!'

Num aut egisse umquam iratum Aesopum aut scripsisse existimas iratum Accium ? Aguntur ista praeclare et ab oratore quidem melius, si modo est orator, quam ab ullo histrione, sed aguntur leniter et mente tranquilla.

Quant à l'orateur, il ne lui sied nullement de se mettre en colère ; le feindre cela ne lui messied pas. Penses-tu que je me mette en colère, toutes les fois qu'il m'arrive dans les procès de hausser le ton et de m'échauffer? Penses-tu que, l'affaire étant jugée, et absolument finie, quand je mets mon discours par écrit, je sois en colère en écrivant?

«Y a-t-il quelqu'un qui le remarque? Attachez-le.»

Crois-tu qu'Ésope fût jamais en colère en jouant ce texte, ou Accius quand il l'écrivit? On peut s'attirer la gloire en déclamant ces vers, et en tant qu'orateur, si l'on est un vrai orateur, plus encore qu'aucun comédien, mais en les jouant tout en restant soi-même calme et plein de sang-froid ${ }^{11}$.

12 Pour un Latin en effet, la littérature est d'abord discours. Qu'on se souvienne de l'éloge cicéronien d'Hortensius (Brut. 301) : 
memoria tanta fuit quantam in nullo cognouisse me arbitror ut, quae secum commentatus esset, ea sine scripto uerbis eisdem redderet quibus cogitauisset. Hoc adiumento ille tanto sic utebatur ut sua et commentata et scripta et omnia aduersariorum dicta meminisset.

Il avait une mémoire si grande que je pense n'avoir connu personne qui l'ait dépassé sur ce point, ce qui faisait que tout ce qu'il avait imaginé intérieurement il le restituait sans l'avoir écrit dans les termes mêmes dans lesquels il l'avait pensé. Il avait recours à un atout si grand pour garder en mémoire à la fois ses idées et ce qu'il avait écrit et tout ce qu'avait dit l'adversaire.

13 Au-delà du tour de force de l'orateur, ce qui retient ici est bien l'ordre des opérations : le discours naît dans sa littérarité avant d'être écrit, et, au fond, le stade de l'écriture ne s'impose pas ${ }^{12}$. Hortensius accède à la littérarité par son discours, et, si son discours devient texte, c'est un heureux concours de circonstances qui conservera, pour ceux qui n'auront pas eu la chance de l'entendre, le discours de l'orateur. Ainsi, le support même de l'écrit qui préside à l'élaboration littéraire - fragile et souvent de piètre valeur : cire, argile ou autre ${ }^{13}$ - souligne face à la dignité du volumen la nature mouvante du discours qui n'est pas encore un texte. À ce titre, la pratique de la recitatio montre la nature mouvante de cette frontière entre discours et texte, presque texte et plus tout à fait discours improvisé ${ }^{14}$. Mais en témoigne également, pour un auteur qui n'est pas un orateur, cette anecdote sur la Vie de Virgile qui ouvre le commentaire de Philargyrius: le poète dictait le matin des vers nombreux ${ }^{15}$ qu'il retouchait toute la journée pour n'en conserver que l'un ou l'autre le soir ${ }^{16}$ :

Et cum Georgica scriberet, traditur cotidie meditatus a mane plurimos versus dictasse solitus ac per totum diem retractando ad paucissimos redigere.

Et dans la rédaction des Géorgiques, il réfléchissait chaque jour le matin, dit-on, et dictait un grand nombre de vers, puis il les retouchait toute la journée et les ramenait à un très petit nombre.

14 La fabrique du texte est donc avant tout une fabrique de discours ${ }^{17}$, ce qui explique par exemple que les commentateurs analysent toute production littéraire d'abord en termes oratoires ${ }^{18}$. Quel que soit finalement le support qui nous le transmet, le texte n'est que la fixation d'un discours ${ }^{19}$, il est toujours en un sens ${ }^{20}$ postérieur à la réflexion sur la littérarité dont il est le produit doublement dérivé (de la réflexion au discours, puis du discours au texte) ${ }^{21}$. Or c'est bien sur ce qu'ils considèrent comme le discours ${ }^{22}$, même s'il est fixé en texte, que les commentateurs analysent la littérarité, comme le montrent clairement les formules qu'ils emploient : bene dixit, bene addidit, bene interrogauit, plus souvent que bene scripsit ${ }^{23}$.

\section{Nature du support et perception textuelle : de la littérarité du discours à celle du texte}

15 À ce point se pose la question du rapport entre discours, texte, support et littérarité. Admettons le point de vue romain: la production littéraire est pensée comme un discours (par exemple, pour reprendre le modèle le plus courant proposé par les grammatici romains, sur le modèle du discours du forum) ${ }^{24}$, puis elle est déposée sur un support (volumen de parchemin ou de papyrus) qui fige le discours en un texte ${ }^{25}$ et permet de le conserver mieux que la mémoire elle-même, au moins à nos yeux ${ }^{26}$. Fautil alors considérer que ce travail de fixation n'est pas sans conséquences sur le discours lui-même, et qu'il se contente de reproduire le discours sous une forme nouvelle? Certainement pas, puisqu'on sait, par exemple pour certains discours de Cicéron, que ce qu'il a fait coucher dans un volumen n'est pas ce qu'il avait dit, et qu'il a donc 
recomposé le discours dans le moment où il a choisi d'en faire un texte ${ }^{27}$. Mais allons plus loin : le support lui-même conditionne d'une certaine manière les transformations que va subir le discours pour devenir texte. Il va évidemment perdre son actio ${ }^{28}$, mais aussi se trouver inscrit dans une forme nouvelle de linéarité. L'utilisation, rare ou inexistante, de la ponctuation dans les exemplaires anciens oblige d'ailleurs les grammatici à reconstruire ce degré minimum de l'actio qu'est l'intonation (interrogative, assertive, mais aussi ironique, emphatique, lente ou rapide, criée ou murmurée, etc.) ${ }^{29}$. Une part importante de la lectio consiste donc à redonner au texte (que l'on lit, rappelons-le, le plus souvent à voix haute ou au moins en marmonnant ${ }^{30}$ ) le statut de discours ${ }^{31}$. De plus, le discours ne répète pas et ne revient pas en arrière ${ }^{32}$, alors que le lecteur de volumen peut dérouler puis enrouler et donc lire plusieurs fois certains passages, en omettre d'autres ${ }^{33}$; mieux encore, il possède un discours peutêtre annoté, ou au moins présenté pour la lecture (fût-elle à voix haute) et non pour la seule audition. Ainsi, des rubriques peuvent lui indiquer où il en est, qui parle, mais aussi où commencent les phrases. Les vers peuvent être soigneusement marqués par des retours à la ligne pour faciliter la juste prononciation rythmique, des éléments de ponctuation peuvent avoir été introduits, etc. ${ }^{34}$. Le scribe lui-même (ou le commanditaire du volumen) a donc superposé au discours premier sa propre vision de la littérarité, comme par exemple en attribuant les répliques ou en introduisant des didascalies entre les scènes dans une comédie ${ }^{35}$, ou en faisant réaliser des peintures pour des scènes particulières ${ }^{36}$. Tout cela relève non du discours lui-même, mais de l'idée de ce discours devenu texte que se fait son premier, deuxième, dixième lecteur, ou copiste ${ }^{37}$. Ce processus a dû donner lieu d'ailleurs à de grandes confusions car, à la fin de l'Antiquité, on voit apparaître des entreprises de réédition des classiques qui visent sans doute à mettre un peu d'ordre dans les dizaines d'exemplaires qui circulent et où chacun y est allé de sa propre annotation ${ }^{38}$. Il en va d'ailleurs de même du texte biblique où les traductions diffèrent, mais où il est aussi devenu parfois difficile de savoir si tel ou tel verset est interrogatif ou affirmatif, s'il complète le précédent ou constitue une nouvelle phrase, etc.

\section{La construction d'une « littérarité seconde »}

Plus en aval, les travaux de Louis Holtz en particulier ont très bien montré que les conditions de passage du volumen au codex au cours des $\mathrm{III}^{\mathrm{e}}-\mathrm{V}^{\mathrm{e}}$ siècles ont accompagné, voire provoqué une certaine sacralisation du texte, annonciatrice de la disparition de tout ce qui n'avait pas eu la chance de mériter le volumen. Dans ce cadre, le critère de sélection a été évidemment posé parfois autrement qu'en termes de littérarité, en termes conservatoires et utilitaires et non littéraires. On comprendrait mal sinon pourquoi on a conservé Pélagonius et pas Gallus, Végèce et pas la fin de Tacite ${ }^{39}$.

On voit alors très clairement qu'il s'opère un déplacement du critère de littérarité. Les textes, conservés évidemment à des fins souvent littéraires, mais aussi parfois purement pédagogiques ou utilitaires, deviennent une sorte de norme de littérarité, quelle que soit la valeur littéraire que nous leur accordons nous aujourd'hui. Nous sommes ici dans une littérarité seconde, surimposée par le choix culturel de copier tel ou tel texte à tel ou tel moment ${ }^{40}$.

18 Toutefois, il ne faudrait pas exclure non plus du champ de la réflexion l'acte même de copie, c'est-à-dire la reproduction du discours devenu texte, et dont toute personne qui 
a déjà eu entre les mains un manuscrit sait bien qu'elle est une entreprise à la fois éditoriale et auctoriale. Toute copie est une lecture, et impose donc des choix au texte qui est copié. Ces choix peuvent être involontaires (fautes de copie), mais ils sont très souvent délibérés et traduisent la vision de la littérarité qui est celle du copiste. Pour les commentateurs, recopier le texte ou simplement les lemmes, marquer ces lemmes, distinguer ce qui est de Donat ou de Servius de ce qui est de Virgile, constitue une première réflexion sur la littérarité, la conscience d'avoir devant soi un discours sur un discours.

Mais cela va évidemment souvent beaucoup plus loin, et il se produit ce que j'appellerais un effet d'«aspiration par la littérarité». Si le copiste voit dans son exemplaire quelque tournure qu'il juge indigne de la grandeur de Servius ou de Virgile, il va avoir tendance à soupçonner son prédécesseur de négligence et à "améliorer » sa copie en corrigeant. De même, tel commentaire redondant ou jugé stupide ne sera pas recopié, «car il ne peut pas être de Donat». On voit bien ici combien finalement la littérarité du texte est aussi affaire de lecture ${ }^{41}$.

Pour prendre un exemple bien plus tardif mais particulièrement révélateur de ce processus de projection par le copiste de sa propre conception de la littérarité : en $P h$. 325, le commentaire de Donat présente de graves désordres, alors que le texte de l'édition de référence (Wessner 1902) semble en apparence excellent. Le voici (325.2) :

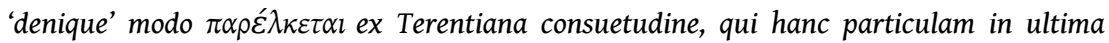
ponit sententia.

Denique est parfois pléonastique selon l'habitude de Térence qui place cette particule en fin d'énoncé.

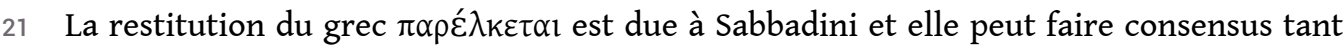
que l'on ne regarde pas les manuscrits. Pour ne prendre qu'un choix limité mais représentatif, $K R C$ lisent par electa ex terentiana, $O$ lit parte lecta ex terentiana; a quant à lui écrit par electa et terentiana, tandis que $G$ présente ici une lacune supposée contenir un mot grec, et seul $V$, qui lit parelceta terentiana, peut accréditer la lecture de Sabbadini. Or plusieurs difficultés se font jour. La première est que si $V$ et $G$ attestent de la présence de grec, l'un par le mot parelceta et l'autre par la lacune, d'autres témoins qui d'ordinaire laissent un blanc sur les mots grecs ne lisent visiblement pas de grec ici. De plus, si l'on observe le vers commenté, uereor, ne istaec fortitudo in neruum erumpat denique, il est difficile de comprendre où se situe exactement le pléonasme. La seule explication possible serait que denique ferait pléonasme avec le sens du verbe erumpat.

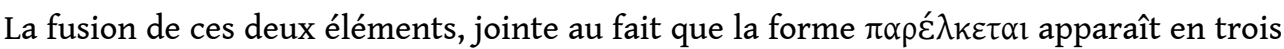
autres lieux du commentaire (An. 579 et $591^{42}$, Eun. 393), laisse supposer que le copiste de $V$, par ailleurs très savant et soigneux comme le montrent de très nombreuses interventions pertinentes de sa main sur des passages fort altérés, a pu imaginer qu'il y avait ici du grec, alors que ses collègues se sont contentés de copier plus ou moins ce qu'ils voyaient ${ }^{43}$. Ainsi, sans exclure tout à fait que $V$ ait eu raison dans son intervention et retrouvé un texte malmené, il est possible également de voir dans cette «correction » la trace d'une lecture savante et attentive, mais aussi tributaire de la connaissance du grec et du commentaire qu'a indéniablement le scribe de $V$, et donc d'une interprétation ${ }^{44}$ de ce qu'il croit être la manière de faire de Donat, accréditée par d'autres passages ${ }^{45}$. 

textuelle parfois difficile à évaluer pour lui. Les remarques ecdotiques peuvent d'ailleurs manifester que le commentateur a une conscience aiguë du fait que ses prédécesseurs ont pu faire des choix textuels en fonction de leur propre perception de ce qu'avait "voulu l'auteur ", autrement dit de leur perception de sa littérarité ${ }^{53}$. Dans le cas d'An. 592, où certains lisent audiam au lieu de audio que Donat a visiblement sur son exemplaire, le commentateur note clairement que le choix de audiam vise à mieux calquer la formule térentienne sur l'original grec de la pièce :

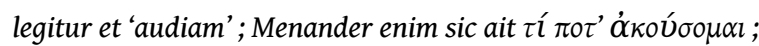




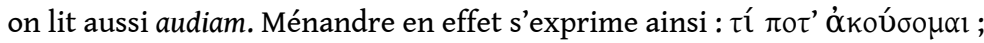
l'on se fait du sens même de telle ou telle réplique. Ainsi en An. 40, Donat propose sur haud muto factum de Térence une variante qu'il analyse en discutant les conséquences littéraires de cette variation :

HAVD M. F. legitur et 'multo', hoc est 'damno', 'reprehendo'. quod si est, sic intellegeretur, 'non nollem factum': nemo enim potest factum infectum reddere. Sed aduerbialiter dixit, ut dicimus "nollem factum ", "nollem exitum ». Ergo 'haud muto factum' 'non me paenitet facti'.

HAVD MVTO FACTVM on lit aussi multo, à savoir 'je condamne', 'je reproche'. Dans ce cas il voudrait dire : «je ne voudrais pas que cela ne fût pas fait » : car nul ne peut défaire ce qui a été fait. Mais il l'emploie adverbialement comme nous disons nollem factum et nollem hunc exitum. Donc haud muto factum 'je ne regrette pas ce que j'ai fait'.

ces deux cas, le commentateur s'inscrit dans une chaîne interprétative dont il a conscience de dépendre, et sa propre lecture se construit de manière manifeste en regard des lectures antérieures. Ce qui affleure ici clairement n'est évidemment pas absent ailleurs, et le genre même du commentaire se pose sans cesse la question de savoir ce qui était l'intention de l'auteur et ce qui est glose surimposée à lui par la tradition.

Mais pour nous modernes, le genre du commentaire est encore plus intrinsèquement médiat car, même si le commentaire est signé (ce qui, rappelons-le, n'est pas un cas constant, les corpus de gloses étant plus ou moins anonymes), il est en fait déjà la compilation par le commentateur de commentaires antérieurs visibles par des mentions du type sic Probus ${ }^{54}$. Ainsi la littérarité qu'il met en évidence dans le texte qu'il commente résulte à la fois d'une réflexion personnelle et d'une tradition interprétative qui a déjà conditionné le texte source dans une certaine approche que le commentateur peut discuter ou contredire mais qui existe comme partie même de sa réflexion, comme dans ces deux exemples de Donat et Servius (Don. Ter., Ad. 323 et Serv., A. 2, 557) :

QVID FESTINAS MI GETA Probus personae assignat hoc Sostratae, Asper non uult ad omnia seruum respondere, sed nutricem putat hoc loqui.

QVID FESTINAS MI GETA Probus attribue la réplique à Sostrata, Asper n'est pas d'avis que l'esclave répond à tout mais pense que c'est la nourrice qui dit cela.

quod autem Donatus dicit, 'litus' locum esse ante aras, a litando dictum [...] ratione caret : nam a litando 'li' brevis est, et stare non potest versus.

Ce que dit Donat, à savoir que le litus est un lieu devant l'autel qui vient de lito, n'a aucun sens; de fait, s'il vient de lito, li est bref et le vers est faux.

31 Ainsi donc la littérarité se présente dans les commentaires au moins sous une quadruple forme : la littérarité du texte commenté, la littérarité que la tradition voit dans le texte commenté, la littérarité que le commentateur voit dans le texte commenté, la littérarité propre du commentateur.

Ici il serait tentant de rejeter tout crédit à la littérarité propre du commentateur, et d'utiliser les commentaires parvenus jusqu'à nous comme de simples documents, témoins de traditions interprétatives, mais sans littérarité propre. Or ce serait se tromper lourdement, car le crédit d'autorité de ces textes les fait accéder à une forme de littérarité qui transparaît dans l'usage intertextuel qui en est fait sur le modèle de la "note alexandrine». Ainsi, quand un auteur tardif cite Virgile, ce n'est pas tant le Mantouan qu'il cite, que «son » Virgile, celui qu'il a étudié, qu'on lui a expliqué, donc

Interférences, 6 | 2012 
celui des commentateurs. Autrement dit, la littérarité qu'il entend chez Virgile passe à travers le filtre d'une lecture qui a déjà souligné tel ou tel point comme remarquable. Je ne prendrai qu'un seul exemple à mon avis très parlant.

$\mathrm{Au}$ début du v $\mathrm{v}^{\mathrm{e}}$ siècle, on trouve, dans le De reditu suo de Rutilius Namatianus, une erreur sur une petite cité côtière du nord de Rome, Castrum, qu'il confond avec Castrum Inui qui se trouve au sud, et il écrit (Red. 227-236):

Stringimus +expugnatum+ et fluctu et tempore Castrum :

Index semiruti porta vetusta loci.

Praesidet exigui formatus imagine saxi, qui pastorali cornua fronte gerit.

Multa licet priscum nomen deleverit aetas, hoc Inui Castrum fama fuisse putat, seu Pan Tyrrhenis mutavit Maenala silvis, sive sinus patrios incola Faunus init; dum renovat largo mortalia semina fetu fingitur in Venerem pronior esse deus.

Nous longeons ensuite Castrum, dont les murs sont rongés par les flots et par les années : une vieille porte indique l'emplacement de cette ville à demi ruinée. Cette porte est gardée par une petite statue représentant un dieu champêtre et par devant portant une inscription. Quoique le temps ait effacé l'ancien nom de cette ville, on dit que c'était le Castrum d'Inuus. Inuus, c'est Pan qui a quitté le Ménale pour les forêts de la Tyrrhénie, ou Faune, qui parcourt encore les lieux de sa naissance ; ce dieu renouvelle toutes choses et verse partout la fécondité ; c'est pour cela qu'on le suppose si ardent pour les plaisirs de l'amour.

Or cette confusion vient d'une lecture de commentateurs virgiliens que l'on trouve chez Servius. Rutilius a pensé aux vers de Virgile pour illustrer son Castrum, mais il a pensé les vers avec le commentaire (faux dans son cas, mais il l'ignore), et le tout a été importé tel quel. Servius commente en effet (Serv., A. 6, 775):

CASTRVMQVE INVI Una est in Italia civitas, quae castrum novum dicitur: de hac autem ait 'castrum Inui', id est Panos, qui illic colitur. Inuus autem latine appellatur, graece П'óv : item

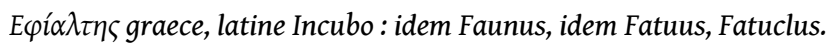

CASTRVMQVE INVI, c'est une cité d'Italie que l'on appelle aussi Castrum Novum; la raison pour laquelle on la nomme Castrum Inui est qu'on y vénère Pan. En effet en latin on l'appelle Inuus, en grec Pan, de même Éphialte en grec se dit en latin Incubo, c'est le même qui est Faunus, Fatuus et Fatuclus.

Ici la littérarité propre du commentateur l'emporte même sur celle de la source, l' auctoritas du grammaticus est telle que sa lecture de Virgile l'emporte sur... Virgile luimême. Ici ce n'est clairement Virgile qui est cité, mais un Virgile commenté que le poète a étudié et qui, dans son esprit, est devenu Virgile.

De la même façon, mais dans un tout autre contexte, preuve de la généralité du processus, Augustin (Conf. 1, 26) reprend, une fois devenu adulte, une célèbre et croustillante scène de l'Eunuque avec le commentaire qui l'accompagnait et qu'on connait par Donat :

ita vero non cognosceremus verba haec, 'imbrem aureum' et 'gremium' et 'fucum' et 'templa caeli' et alia verba quae in eo loco scripta sunt, nisi Terentius induceret nequam adulescentem proponentem sibi Iovem ad exemplum stupri, dum spectat tabulam quandam pictam in pariete ubi inerat pictura haec, Iovem quo pacto Danae misisse aiunt in gremium quondam imbrem aureum, fucum factum mulieri? et vide quemadmodum se concitat ad libidinem quasi caelesti magisterio : 'at quem deum ! inquit...'

Ainsi nous ne connaîtrions plus ces mots imbrem aureum et gremium et fucum et templa caeli et d'autres qui sont écrits dans ce passage si Térence n'avait mis en 
scène un jeune vaurien se proposant Jupiter comme exemple de débauche en regardant une peinture murale où se trouvait la scène où l'on voyait comment Jupiter, selon la légende, a envoyé une pluie d'or dans le sein de Danaé, trompant ainsi cette femme. Et voyez comment il se pousse à satisfaire son désir comme par quelque enseignement du ciel : « Mais quel dieu, se dit-il... »

Don. Ter., Eun. 584, 1 : RESPECTANS TABVLAM QVANDAM PICTAM bene accedit repente pictura ad hortamenta aggrediendae uirginis, ideo quia non ad hoc uenerat Chaerea, ut continuo uitiaret puellam, sed ut uideret, audiret essetque una, cum nihil amplius cogitare ausus fuerit, usque dum picturam cerneret.

RESPECTANS TABVLAM QVANDAM PICTAM il est bien de faire intervenir soudain une peinture pour encourager à agresser la jeune fille, parce que Chéréa n'était pas venu pour immédiatement déshonorer la jeune fille mais pour la voir, l'entendre et être avec elle, vu qu'il n'aurait rien osé imaginer de plus, jusqu'au moment où il a vu la peinture.

Le souvenir d'Augustin est lié au commentaire élogieux que le maître faisait de ce passage en disant que Térence s'y était montré habile, habile, selon le chrétien, à masquer les turpitudes d'une société sous les beautés de la littérature. Dans ces deux cas, il est évident que les auteurs croient citer Virgile et Térence, pourtant ce qu'ils citent n'est pas seulement le classique, mais tout ou partie de sa tradition interprétative. Entre celle de l'hypotexte et celle du texte, le mécanisme de l'intertextualité a glissé une autre littérarité, seconde, celle de la tradition scolaire qui, derrière l'auteur classique, a rajouté un environnement de son cru. De ce fait apparaît immédiatement, avec ces exemples limites, une autre question essentielle, celle de la manière dont la littérarité se fonde sur la notion même d'auctorialité ${ }^{55}$.

\section{Littérarité et auctorialité autour des commentaires}

Le grand nombre de textes anciens dont la littérarité est jugée indéniable, et qui nous sont parvenus sans nom d'auteur assuré ${ }^{56}$, montre que, pour les Anciens en tout cas, littérarité et auctorialité sont deux choses différentes et que l'une n'implique pas nécessairement l'autre ${ }^{57}$. Pour la littérature de commentaire, ce constat a une conséquence bien connue : la manie de l'interpolation, rendue encore plus facile par le fait que le commentaire se présente sous la forme de marginalia, qui sont développables grosso modo tant qu'il reste de la place sur le feuillet ${ }^{58}$. Cette démarche s'accompagne pour nous de son corollaire, la difficulté à déterminer ce qui est interpolé ou non. Car l'interpolateur intervient précisément parce qu'il sent qu'il manque quelque chose à l'original, et, de ce fait, loin d'agir avec désinvolture, il agit par respect pour la littérarité de l'œuvre qu'il interpole. Autrement dit, non seulement il interpole, mais, le plus souvent, il pastiche, c'est-à-dire qu'il reproduit la littérarité même du texte qu'il interpole, non pas tant pour se masquer, mais parce qu'il veut "améliorer » le texte, le compléter en restant dans la manière, dans le ton, dans l'esprit ${ }^{59}$. Le cas du mystérieux interpolateur chrétien de Philargyrius, qui finit d'ailleurs par signer Fatosus, est particulièrement révélateur parce que son travail est sans doute beaucoup plus aisé à repérer pour nous qu'il ne l'était pour ses contemporains. Quand il juge qu'on ne peut décemment commenter les Bucoliques de manière purement profane comme l'avait fait le grammairien que les scribes appellent plus ou moins Philargyrius, il glisse, en pur style philargyrien, sa propre glosula chrétienne qui se fondrait parfaitement à l'ensemble si nous ne savions, nous, que le substrat originel du commentaire philargyrien est très probablement païen. 
On voit ici ce que j'appellerais un phénomène d'«aspiration" lié à la littérarité d'un texte : la littérarité de Virgile a inspiré des commentateurs dont l'auctoritas a à son tour « aspiré » des interpolateurs qui ont décalqué ce qu'ils entendaient de la littérarité des maîtres grammairiens, pour parler à leur tour de la littérarité de Virgile. Fatosus ne s'inspire pas de la littérarité de Virgile, mais de celle de Philargyrius, pour écrire ses propres gloses, il pastiche le maitre pour le rendre plus parfait.

Or la forme même du commentaire s'y prête. S'il est difficile, à moins de faire réaliser une nouvelle copie, d'interpoler directement Virgile, il est facile d'ajouter sur son exemplaire d'un Virgile-Donat par exemple quelques notules nouvelles, peut-être d'ailleurs signées à l'origine, puis fondues dans la masse ${ }^{60} \ldots$ La littérarité du commentaire est celle d'un work in progress, ce qui, par parenthèse, retrouve le sens même de la littérarité romaine dans le discours ${ }^{61}$. Au fur et à mesure de ma lecture, j'ajoute mon propre discours, qui devient texte puis, par mégarde ou à dessein ${ }^{62}$, se range sous l'auctoritas du maître ${ }^{63}$. Or, qui nous prouve que ce procédé évident dans le commentaire n'explique pas également, en particulier pour des textes particulièrement difficiles, la multiplication des variantes (je parle ici de vraies variantes, non d'énormités), souvent trop facilement données à l'impéritie (fréquemment surévaluée) des scribes médiévaux? Ne faut-il pas voir dans ces variantes non pas tant des fautes que des témoins d'une approche différente de la littérarité du texte concerné ${ }^{64}$ ?

Mais revenons à nos commentaires, et à nos Donat, Servius, et autres d'abord écrits sous la forme de mémoires reliés au texte commenté par le système des lemmes, puis présentés sous forme de notes marginales dans ce qui a dû s'appeler à un moment P. Vergili Aeneis cum commentis Aeli Donati aliorumque doctissimorum grammaticorum ${ }^{65}$. À un moment ${ }^{66}$, il s'est opéré un premier choix dont les conséquences sur la littérarité ne peuvent être sous-estimées. En effet, la structure même de la page a été pensée non en fonction du texte commenté, mais en fonction de la taille du commentaire à insérer, ce qui marquait clairement que c'était désormais le commentaire qui primait sur le texte lui-même, par ailleurs sans doute disponible dans d'autres exemplaires ${ }^{67}$. Puis à un autre moment de leur histoire (variable selon les commentaires ${ }^{68}$ ), ces textes ont été copiés comme des textes en soi, indépendamment du texte qu'ils commentaient, et, de P. Vergili Aeneis cum commentis Aeli Donati aliorumque doctissimorum grammaticorum, on est repassé à Aeli Donati grammatici in Terentium. Tous nos commentaires ou presque ont subi ce sort, parfois dans toute leur tradition parfois seulement dans une partie ${ }^{69}$, mais, quoi qu'il en soit, cette mutation est fondamentale pour la question qui nous occupe ici.

41 En effet, en séparant le commentaire du texte commenté, les éditeurs tardo-antiques ou médiévaux altèrent profondément la littérarité du texte qu'ils ont reçu et qui peut être elle-même l'altération de la forme première du texte, dont on a vu qu'elle ne se justifiait que par la mise en rapport de deux discours. On peut même dire qu'ils en changent radicalement le critère de littérarité car, de discours sur un discours, le commentaire devient un texte en soi. On en voit d'ailleurs des traces très claires dans les copies de commentaires ${ }^{70}$.

Par exemple, comme il faut bien quand même que le discours de commentaire s'appuie sur quelque chose, on invente des lemmes, en recopiant un petit morceau du texte original qui rend l'accroche du commentaire, sans doute à l'origine marquée plutôt par un signe ou par la position de la scholie par rapport au texte ${ }^{71}$. Mais, dans les stades ultérieurs de la copie, le plus souvent on ne revient pas au texte original. Si les lemmes ne sont pas clairement identifiés (en capitales, ou rubriqués ou soulignés), ils sont très 
régulièrement confondus avec le commentaire lui-même ${ }^{72}$, cette confusion entraînant un sens nouveau pour l'ensemble du segment (lemme non reconnnu + commentaire) qui se met à exister avec sa logique propre, comme un nouveau fragment de texte désormais totalement autonome de la lettre du texte commenté ${ }^{73}$. L'inverse est également possible, introduisant un niveau supérieur de confusion.

Ainsi, au vers 6 du Phormion, on lit quia numquam insanum scripsit adulescentem, que Donat commente ainsi, selon notre édition :

Scripsit igitur ADVLESCENTVLVM ut comicam personam ostendat et artificiose imminuit adulescentulum, quo magis persona a sublimitate tragica discessisset.

Il a écrit ADVLESCENTVLVM de manière à présenter un personnage comique et il a utilisé avec art le diminutif adulescentulus, afin que le personnage soit davantage éloigné du sublime de la tragédie.

Or, dans l'édition de référence (Wessner 1902), on lisait ceci :

SCRIPSIT legitur et 'fecit'. ADVLESCENTVLVM ut comicam personam ostenderet artificiose.. SCRIPSIT on lit aussi fecit. ADVLESCENTVLVM pour montrer avec art le personnage comique...

Le texte de Wessner paraît plausible, mais il cache en réalité une confusion entre lemme et scholie opérée par une partie de la tradition. Le texte fecit n'est attesté par aucun manuscrit de Térence, ce qui est relativement anormal, car les variantes que fournit Donat se retrouvent généralement dans la tradition de Térence. Deux manuscrits souvent très proches l'un de l'autre, $C$ et $O$, lisent chacun igitur et fecit, leçon que l'on pourrait tenir pour l'une de leurs nombreuses bévues, si le texte ne se comprenait pas beaucoup mieux avec igitur qu'avec legitur, si l'on considère que et fecit est le résultat d'une "aspiration d'auctorialité ». En An. 3, Donat a dit en effet QVAS FECISSET FABVLAS bene 'fecisset', non 'scripsisset'. unde et poetae a faciendo dicti sunt, $\dot{\alpha} \pi \dot{o}$ tov

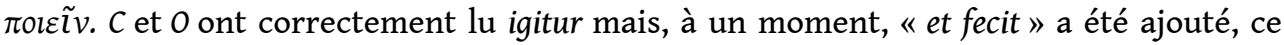
qui s'explique par le fait que scripsit a été pris pour le lemme - puisque la forme figure chez Térence - et qu'il a fallu lui trouver un commentaire qui, en tout état de cause, était igitur. On a donc créé une scholie habituelle avec legitur et à la place de igitur et on a repris, pour compléter, l'idée contenue dans An. 3. Ce qui a provoqué ce désordre ici est que le lemme n'est pas le premier mot de la scholie, alors que tout porte à le croire et que la littérarité propre du genre conduit à cette idée qu'il faut d'abord un lemme puis une scholie.

Ainsi, dans ces cas de copies indépendantes du texte, qui sont les plus fréquents pour les commentaires les plus célèbres, le commentaire lui-même a été envisagé dans sa littérarité propre, comme s'il était une œuvre à part, digne en elle-même d'être étudiée. Ce faisant, on voit qu'il change indéniablement de statut, en particulier en ce qu'il devient modèle, ou texte de référence, pour la production de nouveaux textes.

Toutefois, il ne s'agit sans doute pas que d'un mécanisme lié à la transmission, mais la littérarité même du commentaire, c'est-à-dire ce qui constitue le commentaire en genre littéraire, peut s'y cacher sous une forme que les commentaires chrétiens peuvent nous faire comprendre. En même temps, l'usage chrétien du commentaire peut également sans doute expliquer parallèlement l'évolution de la reproduction des commentaires profanes, par une sorte de contagion de la littérarité des uns sur les autres.

Fondamentalement, le commentaire chrétien et le commentaire profane grammatical reposent sur un discours du même type: une personne pourvue d'une auctoritas 
explique, pour un public supposé moins habile, un texte donné. L'affirmation de l'autorité est d'ailleurs explicite dans la plupart de ces textes, parfois sous une forme directe ( je parle parce que...»), parfois aussi sous une forme indirecte ( je me fais l'écho de l'autorité de...»). Qu'il soit évêque ou grammairien (l'évêque est d'ailleurs souvent un ex-grammairien) ne change fondamentalement rien à l'affaire. Ce qui change en revanche, c'est ce qui s'est produit après, dans le passage du discours au texte. On ne sait pas si quelqu'un a retranscrit un jour les cours de Donat, mais on a retranscrit les sermons des Pères ${ }^{74}$, fixant ainsi en texte un discours de commentaire. On a donc l'impression que, d'emblée, puisque les commentateurs profanes n'ont pas eu cette chance et que leurs cours ont été démembrés en gloses marginales, le statut accordé à leur littérarité était forcément inférieur. Cela est sans doute vrai, mais seulement jusqu'à un certain point de la tradition du texte, et l'évolution perceptible dans certains commentaires chrétiens rappelle, par la bande mais de façon significative, le passage des gloses au texte continu et donc le changement de littérarité accordé au commentaire profane ${ }^{75}$.

Dans le cas d'un texte comme le Commentaire du psautier de Cassiodore, on est absolument certain qu'il ne s'agit pas d'homélies, mais bel et bien d'un commentaire continu rédigé comme tel, dans des circonstances d'ailleurs assez bien connues ${ }^{76}$. Pourtant dans sa structure même, avec ses oraisons conclusives par exemple, et dans l'abondance de l'interlocution, ce commentaire d'un côté affecte le ton de l'homélie, de l'autre le fait glisser vers un autre type d'ouvrage qui s'apparente nettement à une monographie. S'il avoue en effet sa dette envers Augustin (Cassiod., Psalm. Praef.) :

Repulsis aliquando in Rauennati urbe sollicitudinibus dignitatum et curis saecularibus noxio sapore conditis, cum psalterii caelestis animarum mella gustassem, id quod solent desiderantes efficere, auidus me perscrutator immersi, ut dicta salutaria suauiter imbiberem post amarissimas actiones. Sed familiaris incohantibus occurrit obscuritas, quae uariis est intexta personis et uelata parabolis. Haec in dictis uitalibus noxia dissimulatione praeteritur, dum saepe illud reperiri solet ambiguum, quod magni sacramenti gestat arcanum. Tunc ad Augustini facundissimi patris confugi opinatissimam lectionem, in qua tanta erat copia congesta dictorum, ut retineri uix possit relectum quod abunde uidetur expositum. Credo, cum nimis auidos populos ecclesiasticis dapibus explere cupit, necessario fluenta tam magnae praedicationis emanauit. Quocirca memor infirmitatis meae, mare ipsius quorumdam psalmorum fontibus profusum, diuina misericordia largiente, in riuulos uadosos compendiosa breuitate deduxi, uno codice tam diffusa complectens, quae ille in decadas quindecim mirabiliter explicauit.

Quand, il y a un moment, j'eus repoussé à Ravenne les inquiétudes que causaient les charges et les soucis de ce monde qu'accompagne une saveur coupable, comme j'avais goûté ce miel des âmes qu'est le saint psautier, ainsi qu'ont coutume de le faire ceux qui sont pleins de désir, je me plongeai avec avidité dans la recherche, afin de m'imprégner de la douceur de ces paroles salutaires à la suite d'actions qui m'avaient causé une particulière amertume. Mais, la compagne qui surgit aussitôt aux débutants, c'est l'obscurité, qui se glisse dans les divers personnages et se cache dans les paraboles. Ces éléments, il y aurait une dissimulation coupable à les laisser de côté dans des paroles aussi vitales, alors que souvent ce que l'on trouve ambigu, c'est ce qui porte le secret d'un grand mystère. Alors je me réfugiai dans la lecture la plus assidue de notre très éloquent père Augustin ; il s'y trouve une telle masse et abondance de paroles que c'est à peine si on peut retenir en relisant ce qui nous semble avoir été abondamment commenté. Je crois que, en désirant assouvir des festins de l'Église des publics excessivement avides, il est inévitable qu'il ait fait couler à flots cette si grande prédication. C'est pourquoi, me souvenant de ma faiblesse, j'ai réduit, grâce au secours de la divine miséricorde, et en usant de la brièveté que l'on trouve dans les résumés, la mer qui chez lui débordait à profusion 
de la source de certains psaumes aux dimensions de petits ruisseaux aisément traversables, en embrassant en un seul livre des matières si vastes, qu'il lui a fallu quinze décades, chiffre incroyable, pour les commenter.

il présente son propre ouvrage comme une sorte de mise en forme de l'essentiel du commentaire augustinien, conformément à un plan jugé pédagogiquement pertinent, mais aussi détaché de la fonction même de la prédication (Cassiod., Psalm. Praef. 14) :

Primo nobis de titulorum inscriptione, domino iuuante, dicendum est, unde uelut uberibus expressis lacteus sensus diuinae praedicationis emanet. Tum : unusquisque psalmus pro sua qualitate diuidendus est, ne nobis intellectum permisceat aut occulta mutatio rerum, aut uarietas introducta loquentium. Tertio: arcanum psalmi, partim secundum spiritalem intellegentiam, partim secundum historicam lectionem, partim secundum mysticum sensum, subtilitates rerum discutiens proprietatesque uerborum, prout concessum fuerit, conabor aperire. Quarto: prout locus exegerit, uirtutem eius breuiter demonstrare contendam, quatenus intentio digesti carminis diuino munere oculis interioribus elucescat. [...] Quinto: de ordine numeri psalmorum, cum res exegerit, memorabimus, qui reuerendissimarum rerum honore sacratus est. [...] In conclusionibus uero aut summam totius psalmi sub breuitate complectimur, aut contra haereses aliquid dicimus destruendas. Il nous faut d'abord parler, avec l'aide du Seigneur, du titre du psaume, d'où découle comme du lait que l'on tire d'une mamelle le sens de la prédication divine. Puis, il faut pour chaque psaume établir son plan en fonction de sa nature, afin qu'une modification implicite du matériau ne nous échappe pas, ou de même un changement de locuteur. Troisièmement, je tenterai de révéler les secrets du psaume, en partie selon le sens spirituel, en partie selon la lecture historique, en partie selon le sens mystique, en examinant avec soin les subtilités du fond et la propriété des termes, selon la mesure de ce qui me sera donné. Quatrièmement, quand le lieu le demandera, je m'efforcerai d'en montrer la valeur en quelques mots pour que le dessein des parties du poème vienne frapper le regard des yeux intérieurs du don divin. Cinquièmement, quand la situation l'exigera, nous ferons un rappel sur le numéro du psaume, qui est souvent consacré par la dignité de réalités absolument vénérables. Dans les conclusions, soit nous faisons un résumé du contenu de tout le psaume, ou nous disons quelque chose qui vise à détruire les hérésies.

50 Il faut donc comprendre qu'une certaine forme de littérarité s'est trouvée attachée à ce type de commentaire, au point de devenir comme une marque générique qui s'accompagne d'ailleurs d'une référence explicite à une norme, dans le cas de Cassiodore ou d'Augustin, puis, qu'à partir de cette norme nouvelle, il se crée de nouvelles formes qui se fondent sur les critères de littérarité tirés du texte devenu modèle.

51 Pour les commentaires profanes, on peut aisément saisir le même mécanisme dans le moment où le commentaire s'autonomise du texte de départ. Ainsi, le mode même du commentaire séparé du texte commenté aboutit à des textes pensés comme indépendants et conçus dès le départ comme existant sans le texte commenté luimême, au moins dans son intégralité, comme le seront ensuite les annotations médiévales telles que celle de Rémi d'Auxerre sur Martianus Capella ou de Guillaume de Conches sur Boèce et les recueils d'animadversiones, avatar humaniste de ce qu'ils lisaient dans leurs manuscrits de Donat ou de Servius ${ }^{77}$. Cela veut dire que des gloses marginales ont fini par se constituer sous la forme du texte continu lemmatisé comme un texte pourvu de sa propre littérarité, comme un texte modèle, porteur de valeurs formelles propres. 


\section{En guise de conclusion, « mille-feuilles » de la littérarité et édition numérique : la représentation de littérarités successives}

On voit donc ici, avec l'exemple certes particulier du commentaire, comment en réalité la question de la littérarité peut et peut-être doit se poser d'une double manière s'agissant de textes anciens : elle ne peut être envisagée en effet que sur un plan à la fois synchronique et diachronique et elle passe à la fois par la lettre du texte et par les réseaux de son interprétation qui peuvent ensuite passer pour la lettre elle-même. Car, si utile et profitable que puisse être la réflexion sur la littérarité de l'objet premier, disons Virgile, elle ne peut être dissociée de la réflexion connexe sur ce que nous lisons dans Virgile et pourquoi nous le lisons, autrement dit sur les multiples Virgile qui séparent et en même temps approchent de nous le Mantouan ${ }^{78}$. Or c'est à ce point qu'intervient la question des rapports entre l'édition et le mode d'appréhension de la littérarité et que revient sur la table le débat liminaire sur l'évolution des supports. Car si l'on pose la question du rapport entre l'édition et la littérarité, vient alors la question centrale : comment une édition traduit-elle la/les littérarité(s) et la/les traduit-elle?

La question devient alors: qu'éditons-nous et en quoi notre perception même de la littérarité constitue-t-elle un travail d'édition? C'est en fait autour de ce débat que peuvent dialoguer le livre et les supports numériques, non pas en tant que concurrents mais en tant que moyens de montrer des choses différentes et donc d'approcher la littérarité sur un mode différent ${ }^{79}$.

Quel que soit le degré de détail de l'apparat critique ou de l'annotation, une édition livresque n'échappe jamais à une réalité inhérente au support même. Elle représente une lecture synchronique du texte, Virgile à un temps $t$, et on peine à discerner dans l'édition la part de diachronie qui y est implicitement présente ${ }^{80}$.

Se pose alors la question de la place de la diachronie dans la perception de la littérarité. Si elle est faible ${ }^{81}$, le livre est un moyen simple de la représenter, si l'on se souvient très précisément de ce qu'il représente exactement, c'est-à-dire la dernière feuille du «mille-feuilles» de la littérarité. La question se pose évidemment autrement pour des textes dans lesquels la diachronie joue un rôle essentiel dans la perception de la littérarité, comme les commentaires antiques ou encore des textes comme la glose ordinaire, mais également pour les textes qui ont été abondamment glosés car leur littérarité passe aussi, comme on l'a vu, par une histoire de leur lecture, dont la nôtre n'est que la dernière, jusqu'à la suivante ${ }^{82}$.

Or, justement, l'énorme avantage de l'outil numérique consiste en la possibilité d'allier une lecture synchronique et une véritable lecture diachronique, précisément par la possibilité de séparer les feuilles du mille-feuilles soit pour les réordonner dans une autre disposition soit pour les regarder en elles-mêmes, soit encore pour les mettre toutes en rapport sur un critère précis.

Dans le cas précis du commentaire de Donat, on peut considérer que l'édition Wessner (1902), revue par nos soins en fonction des travaux parus depuis, constitue ce que nous appelons un texte pivot, autour duquel gravitent deux types d'activités qui interrogent chacune à leur manière la littérarité du commentaire. D'un côté les divers manuscrits dont nous donnons une collation semi-diplomatique sont pensés non pas comme des témoins de ce texte, mais comme des versions de celui-ci. Cette représentation nous 
permet de projeter sur les collations manuscrites les éléments de structuration moderne du texte, ce qui autorise la parallélisation absolue de chaque segment: le lecteur peut donc voir non pas seulement les variantes de chaque segment (par exemple la seconde scholie au vers $x$ de l'Eunuque), mais bien le texte complet de chaque manuscrit, et aussi choisir d'afficher ou de masquer tel ou tel élément en s'inspirant d'un modèle développé pour des sagas islandaises par l'équipe de M.J. Driscoll ${ }^{83}$. Cette mise en parallèle des versions manuscrites (et non des seules leçons) permet de considérer chaque manuscrit comme un témoin de l'évolution de la lecture du commentaire, mais aussi bien évidemment de le traiter dans la perspective d'une édition critique ${ }^{84}$. Pour la tradition de Donat où deux des principaux manuscrits à l'origine de toute la tradition ont disparu, il est tentant d'utiliser cette méthode pour recouper les textes des manuscrits conservés et envisager quelle proportion des manuscrits perdus est reconstructible et selon quelles modalités. Ainsi le lecteur peut soit s'intéresser à la construction moderne d'un texte de Donat qui repose sur nos connaissances certes, mais aussi sur notre vision de la littérarité de cet ouvrage, soit encore construire sa propre sélection de manuscrits comme autant de témoins de la manière dont on a lu et compris Donat. On mesure évidemment l'avantage supplémentaire qu'apporte cette méthode pour des textes dont la tradition manuscrite s'étend sur plus de dix siècles ${ }^{85}$, et la liberté qu'il donne au lecteur dans sa propre appréhension de ce qu'est le texte, ou de ce que sont les textes qui nous transmettent ce texte.

Pour dire les choses autrement, l'outil numérique, s'il est bien conçu, écrit autant de livres qu'il y a de lectures et de lecteurs. Est-ce à dire que tout schéma de lecture est pertinent et que tout se vaut? Évidemment non, car l'outil ne peut exister sans un moyen d'évaluer sa propre lecture: si je choisis de privilégier telle version du texte, venue de tel endroit à tel moment, l'outil doit me dire quelles en sont les conséquences immédiates sur le reste du texte. Si je choisis toujours la leçon de tel manuscrit par exemple, à quel moment vais-je me retrouver avec un texte qui sera en contradiction avec d'autres choix que j'ai faits, par exemple celui de privilégier les branches insulaires.

59 Un autre avantage de l'outil numérique est qu'il offre une possibilité de prendre vraiment en compte le support, c'est-à-dire la part de littérarité qui se glisse entre le discours et le texte, et qui n'appartient plus au discours, mais déjà à la forme texte ellemême. Nous avons vu que, dans le cas des changements de titre, s'opérait un changement de la perception même de la littérarité du texte, mais d'autres phénomènes jouent, comme par exemple le fait d'écrire les vers en vers et non comme de la prose, d'orner ou de ne pas orner. Si l'on prend le cas de Donat, il est évident que les apographes réalisés par des humanistes pour leurs propres recherches ne disent pas la même chose de la littérarité du texte qu'un magnifique exemplaire comme celui de la bibliothèque de Cesena où l'œuvre est placée dans un écrin qui à lui seul atteste de l' auctoritas qu'elle possède ${ }^{86}$.

60 Évidemment, me dira-t-on, tout cela n'est qu'interprétation et n'affecte pas la littérarité originelle, celle que nous traquons. Ce serait vrai si nous étions certains que ce que nous lisons est bien ce qui a été écrit par Virgile, Homère ou Pindare, si nous avions ce contact immédiat avec le texte, et non avec des copies, bref si nous n'avions pas ou plus besoin d'éditer ${ }^{87}$. Or ce n'est pas le cas et ce ne peut être le cas. Au mieux, pour reprendre les paroles de L. Holtz, lisons-nous ce que lisaient les lecteurs du IV 
siècle ${ }^{88}$. La question est donc alors : quelle influence sur notre vision de la littérarité a cette histoire de la transmission ou, plus exactement, avons-nous conscience que les modalités mêmes de la transmission des textes, jusque dans leurs aspects les plus concrets, peuvent influer sur notre vision de leur littérarité?

Cette question rebondit d'ailleurs sur une autre qui est particulièrement vive quand on prépare pour l'édition numérique des textes à la tradition manuscrite extrêmement confuse comme le commentaire dit de Philargyrius. N'est-il pas au moins aussi important pour la compréhension du texte lui-même de savoir comment il a été lu, tronqué, augmenté, bouleversé, que de disposer d'un texte qui, malgré tous nos efforts de reconstitution, sera finalement assez arbitraire. Dans un cas comme celui-ci, mais qui vaut pour la plupart des commentaires dits grammaticaux, c'est finalement le mille-feuilles tout entier qui porte la littérarité, et non telle ou telle feuille. Sur ce point qui est acquis pour tous les lecteurs de commentaires antiques, il est évident que l'étude des commentaires a beaucoup à apporter au reste de la philologie, au moins pour rendre plus constante l'attention aux diverses strates qu'a traversées tout texte ancien. Finalement sans doute, une édition numérique de Virgile sur les mêmes bases que celle que nous préparons pour Donat donnerait sans doute matière à renouveler considérablement notre vision du Mantouan dans les divers avatars qu'il a connus au fil des siècles.

Et on en revient alors à ce que nous citions au début: "dans le moment actuel de basculement et de crise culturelle, la disparition annoncée du livre de papier au profit des multiples formes de l'électronique n'apparaît pas comme le passage d'un support à un autre, et comme la simple évolution d'un vecteur, mais comme la transformation radicale de la notion même de texte ». Il ne s'agit sans doute pas pour nous de transformer la notion de texte, ni surtout d'entrer dans un conflit (suicidaire pour nous) avec le livre, mais bien de montrer par cet exemple des commentaires, évidemment choisi parce qu'il montre plus clairement que bien d'autres textes quels sont les enjeux, comment l'outil numérique questionne non pas tant le livre, ou même le texte, que nos modes habituels de perception de la littérarité. L'outil numérique, parce qu'il questionne notre lecture et le mode même de la lecture, ne ferme pas la porte à la littérarité, il ouvre en réalité des fenêtres sur des formes jusqu'ici restées peut-être plus dans l'ombre de l'analyse littéraire.

\section{BIBLIOGRAPHIE}

BUREAU B., NICOLAS C. (éds) 2010, HyperDonat : une édition électronique des commentaires de Donat aux comédies de Térence, Lyon [en ligne]. Disponible sur : <http://hyperdonat.ens-lyon.fr/> (consulté le 11.05.2012).

DORANDI T. 2000, Le stylet et la tablette: dans le secret des auteurs antiques, L'Âne d'or 12, Paris.

FUNAIOLI G. 1930, Esegesi virgiliana antica: prolegomeni alla edizione del commento di Giunio Filargirio e di Tito Gallo, Annali della Facoltà di Agraria - Università Cattolica del Sacro Cuore. Milano 9, Milan. 
GURD S. 2007, «Cicero and Editorial Revision », ClAnt 26, 1, p. 49-80.

HABINEK T. 2005, « Latin Literature between Text and Practice », TAPhA 135, 1, p. 83-89.

HOLTZ L. 1984, « Les manuscrits latins à gloses et à commentaires de l'Antiquité à l'époque carolingienne », in C. Questa, R. Raffaelli (éds), Atti del Convegno internazionale «Il libro e il testo»: Urbino, 20-23 settembre 1982, Pubblicazioni dell'Università di Urbino. Science umane 1, Urbino, p. $141-166$.

HOLTZ L. 2000, « Le rôle des commentaires d'auteurs classiques dans l'émergence d'une mise en page associant texte et commentaire (Moyen-Âge occidendal) ", in M.-O. Goulet-Cazé et al. (éds), Le commentaire : entre tradition et innovation, Actes du colloque international de l'Institut des traditions textuelles (Paris et Villejuif, 22-25 septembre 1999), Bibliothèque d'histoire de la philosophie, Paris, p. 101-118.

KER J. 2004, « Nocturnal Writers in Imperial Rome: the Culture of Lucubratio », CPh 99, 3, p. 209-242.

LEDENTU M. 2004, Studium scribendi : recherches sur les statuts de l'écrivain et de l'écriture à Rome à la fin de la République, Bibliothèque d'études classiques 39 , Louvain.

MAEHLER H. 2000, «L'évolution matérielle des hypomnèma jusqu'à la basse époque. Le cas du POxy. 856 (Aristophane) et PWürzburg 1 (Euripide) ", in M.-O. Goulet-Cazé et al. (éds), Le commentaire : entre tradition et innovation, Actes du colloque international de l'Institut des traditions textuelles (Paris et Villejuif, 22-25 septembre 1999), Bibliothèque d'histoire de la philosophie, Paris, p. 101-118.

MCDONNELL M. 1996, «Writing, copying, and autograph manuscripts in ancient Rome », CQ 46, 2, p. 469-491.

MARSHALL A.J. 1976, « Library Resources and Creative Writing at Rome », Phoenix 30, 3, p. 252-264. MOUNIER P. 2011a, «Qu'apportent les digital humanities? Quelques exemples (1/2)», Homo Numericus [en ligne]. Disponible sur : <http://www.homo-numericus.net/spip.php?breve1011> (consulté le 11.05.2012).

MOUNIER P. 2011b, «Qu'apportent les digital humanities? Quelques exemples (2/2) », Homo Numericus [en ligne]. Disponible sur : <http://www.homo-numericus.net/spip.php?breve1012> (consulté le 11.05.2012).

O’DONNELL J.J. 1979, Cassiodorus, Berkeley.

ROBINSON P. 2004, « Where we are with Electronic Scholarly Editions, and where we want to be » [en ligne]. Disponible sur : <http://computerphilologie.uni-muenchen.de/jg03/robinson.html> (consulté le 11.05.2012).

TURA A. 2005, «Essai sur les marginalia en tant que pratique et documents », in D. Jacquart, C. Burnett (éds), Scientia in margine : études sur les Marginalia dans les manuscrits scientifiques du Moyen Âge à la Renaissance, Hautes études médiévales et modernes 88, Genève, p. 261-387.

VALETTE-CAGNAC E. 1997, La lecture à Rome : rites et pratiques, L'Antiquité au présent, Paris.

\section{NOTES}

1. Colloque du GDRI CLARo, "Avènement et reconnaissance de la littérarité dans l'Antiquité romaine », université Paris Ouest, 18-19 novembre 2011. 
2. Voir Mounier 2011a et surtout 2011b dont nous extrayons cette très éclairante réflexion:

«Mais peut-être faut-il aussi y voir la conséquence d'une évolution significative des conditions de communication des résultats de recherche qui doivent être plus rapidement accessibles et selon des formes plus courtes. Dans bien des cas, le passage de l'imprimé au numérique s'est traduit pour l'édition de sciences humaines et sociales par la montée en puissance de la forme article ou contribution à des ouvrages collectifs contre la prééminence de la monographie, puis par l'apparition de formes 'très courtes' comme le billet de blog dont certains affirment qu'il est appelé à remplacer à son tour l'article devenu obsolète.

On le voit bien, l'arsenal théorique dont Bourdieu s'équipe pour explorer le monde social est difficilement présentable sous forme de billets de blog. Comme on le sait, la forme et le fond sont indissociables. C'est ici le livre dans sa forme la plus traditionnelle qui est le plus adapté à l'exposition d'une théorie sociologique qui n'appartient qu'à son auteur et dont la sophistication a besoin de temps et de place pour s'exposer. Lorsqu'il entreprend sa critique en profondeur de La Distinction, ce n'est ni un site web que propose Bernard Lahire, ni une base de données en ligne ou un graphe social, encore moins un carnet de recherche ou une bibliographie collaborative, mais un ouvrage de presque 800 pages, La Culture des individus, dont la réalisation et la composition ne doivent pas grand-chose à la mobilisation des technologies numériques.

Si les promesses des digital humanities provoquent un enthousiasme justifié, les sciences humaines et sociales au prisme du numérique semblent, elles, écartelées entre plusieurs pôles qui peuvent se révéler comme autant d'écueils: d'un côté la fascination pour l'outil qui peut conduire, dans le cas très fréquent des corpus instrumentés, à des jeux non contrôlés dont la rigueur et la pertinence n'est pas toujours évidente; de l'autre, l'adoption de formes d'interventions plus réactives et plus fluides, mais aussi plus fragmentées dont le pouvoir critique risque d'être très affaibli par le cadre médiatique dans lequel il s'insère. Enfin, des constructions théoriques fortes, mais dont le mode d'expression risque de se marginaliser fortement et de perdre de son efficacité parce que très éloigné des lieux et des formes d'échanges très contemporains. "

Venant d'un acteur et d'un observateur majeur des activités d'édition numérique dans notre pays, ce constat appelle évidemment toute l'attention des éditeurs numériques que nous sommes, car il interroge à la fois notre mode de lecture et notre mode d'écriture dite «scientifique».

3. Voir à ce sujet Robinson 2004 qui développe une vue optimiste des rapports entre le philologue et l'outil numérique dans l'optique de la création d'une nouvelle communauté humaniste, fondée sur un dialogue des savants rendu bien plus facile par l'outil numérique. L'édition critique du commentaire de Donat aux comédies de Térence que nous entamons pour développer notre réalisation Bureau, Nicolas (éds) 2010 représente une forme de cette avancée vers une « communauté éditoriale » là où notre pratique habituelle est celle d'un philologue isolé, voir à ce sujet le billet «Appel à collaboration » (http://hyperdonat.hypotheses.org/249) sur le carnet de recherche en ligne de nos travaux.

4. Voir ici encore Robinson 2004 qui pose cette question de manière particulièrement pertinente du point de vue du "philologue numérique ».

5. Par ex., Don. Ter., Eu. 9 : Et bene NVNC NVPER, ut ex uicinitate facti ostendat nihil esse dicendum, quam displicuerit haec comoedia Luscii Lanuuini, propterea quod res recens sit et omnes meminerint («Et NVNC NVPER est bon pour montrer qu'en raison de la proximité de l'événement il n'y a rien à ajouter pour dire combien la comédie de Luscius Lanuvinus a déplu, puisque la chose est récente et que tout le monde s'en souvient »); Don. Ter., Ph. 1005 (avec une discussion sur la pertinence de l'analyse) : мі номо DI MELIVS DVINT quaerit Probus, an matrona tam familiariter recte dicat alieno, sed frustra: nam feminarum oratio, etsi non blanditur, blanda est (« MI HOMO DI MELIVS DVINT Probus se demande s'il est juste qu'une femme romaine s'adresse de façon si intime à quelqu'un d'autre, mais c'est en vain, car le propos d'une femme, même si on n'est pas doux avec elle, est doux »). 


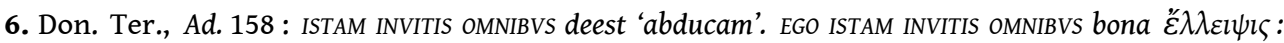
nemo enim plane loquitur, qui luctatur (« EGO ISTAM INVITIS OMNIBVs bonne ellipse ( $\left.{ }^{\prime} \lambda \lambda \varepsilon \mid \psi 1 \varsigma\right)$ : car personne ne fait des phrases complètes dans un affrontement »).

7. Don. Ter., Hec. 1 : HECYRA EST HVIC NOMEN FABVLAE nominatiuo casu figurauit, cum in usu sit, ut datiuo dicamus. quamuis praesto sint exempla, quibus ueteres per omnes fere casus hoc genus locutionis enuntiabant ( HECYRA EST HVIC NOMEN FABVLAE l'auteur a fait une figure en mettant le titre au nominatif quand selon l'usage nous mettrions le datif. On a cependant des exemples à disposition qui montrent que les Anciens mettaient ce genre d'énoncé à presque tous les cas »).

8. Voir sur ce point, dans le présent numéro, l'article de Marcos Martinho qui montre la complexité des approches du phénomène littéraire autour de la question de ce qui est ou non poétique.

9. Il faut les dissocier, non pour les opposer, mais pour mieux percevoir le type particulier de relation qui unit l'un à l'autre. Voir Habinek 2005, p. 86: "The problematical relationship between embodied performance and textual symbolization is at the heart of Latin literature. Which controls which? [...] It is precisely because Latin literature is both oral and literate, both textual and embodied, both intimate and spectacular that it constitutes such a valuable case study of relations between mimesis and semiosis. Moreover, the tactics that were used by certain sectors of Roman society to organize the mimetic and semiotic potential of Latin literature have provided influential models for successive waves of organization -right on up to the present. »

10. Voir aussi Brut. 91 qui atteste que c'est la pratique courante pour les orateurs. Pour Cicéron lui-même, il existe de multiples témoignages bien connus, en particulier à propos des deux versions du Pro Milone.

11. Précisément parce que, pour contrôler exactement l'effet que l'on donnera à ces vers, il faut savoir jouer sur la conscience que l'on a de soi-même en tant qu'acteur. On est ici dans le paradoxe du comédien, qui est aussi en un sens le paradoxe du discours et du texte.

12. Cicéron peut d'ailleurs déplorer la disparition de certains discours tout simplement parce que leur auteur ne les écrivait pas, bien que ce fait reste exceptionnel. McDonnell 1996, p. 476 : «In explaining why copies of the speeches of Publius Sulpicius did not survive, Cicero pointed to the orator not having been accustomed nor able to write them; the implication being that other orators could and did".

13. Sur ce point, voir entre autres McDonnell 1996, p. 473.

14. Ker 2004, p. 210, rappelle: "As it is described in the letters of Pliny the Younger, the recitation involved a reading out loud of the written but as yet unpublished text (whether by the author or by an assistant, with the author close by) in the semipublic interior space of an auditorium or house to which a small audience had been invited. It thus constituted a first, oral publication of the text, and its conditions could greatly influence the text's reception. It created an opportunity for evaluation, for example, in the care taken by the author to invite an audience who would affirm the social value (dignitas) of the occasion and hence of his text, and to avoid the "chill" (frigus) that could result from inappropriate behavior or an unsympathetic response; or in the audience's expression of judgment (iudicium) through subtle gestures, which could result in the author making emendations before the next phase of wider publication. The recitatio was thus also characterized by a spirit of mutual exchange or reciprocity (gratia), and we find that to attend a trying daylong recitatio required one to curb one's autonomy (libertas) in the service of generosity (liberalitas); in some cases this was justified by the observation that someone who is in the present instance a listener (auditor) will at some later date be a performer (recitator) and thus dependent on the generosity of his listeners in turn ».

15. Sur le lien entre dictée et écriture, voir McDonnell 1996, p. 474 : «Authors of speeches and literary works often dictated to slaves, the practice being perhaps more regular among prose authors than poets. But as Horsfall's nuanced study pointed out, the place of dictation in composition was complex, and an author might dictate a text after he had written it up himself. 
Pliny's correspondent, Voconius Romanus, stated explicitly that he was both dictating and writing a literary composition: 'multa te nunc dictare, nunc scribere' (Ep. 9.28.3). Other authors composed their works sua manu by choice. It is difficult to know how common the preference for writing by hand was, but a general connection between literary composition and writing sua manu in the late Republic is suggested by the fact that when Cicero wished to have the interrogation of the Catilinarian conspirators recorded, and required senators whose handwriting he knew to be practiced and fast, he selected literary men for the job (Sull. 42)».

16. Il est évident qu'il ne faut pas accorder à ce texte trop de valeur historique, mais peu importe ici. Ce qui compte, c'est de voir comment le rédacteur se représente le processus idéal de création du plus grand des poètes.

17. Le discours préexiste à toute composition, y compris textuelle, si l'on en croit le conseil donné par Fortunatianus, 3, 159, 8 cité par Valette-Cagnac 1997, p. 33 : et priusquam scribere incipiamus, ut animus legendo calescat, legemus aliquid uel cum tenui murmure (« et avant de commencer à écrire, pour nous échauffer l'esprit par la lecture, nous lirons un passage, ne seraitce que dans un petit murmure »).

18. Ainsi Donat analyse souvent comme des controverses ou des suasoires les scènes comiques térentiennes, comme par ex. Don. Ter., Eu.666, 3 : Et iam si penitus consideraueris, controuersia inerit: si enim nihil posse audieras, quid est quod in mentem non uenerit ut uitares? utrum igitur 'amatores mulierum esse maximos' audieras? quod ipsum uitandum fuit; neque enim hoc satis est ad pudicitiam stuprum uitasse, cum impudica fieri uel solo osculo possit, quam omni modo integram et inlibatam uelis. an 'uerum' non erit coniunctio sed nomen, ut illud quasi falsum audierit, hoc autem quod uerum inuentum est, non suspicata sit? nam 'in mentem uenire' non 'reminisci' tantum, sed etiam 'cogitare' significat («Et maintenant, si vous examinez ce texte à fond, il y aura controverse. Car même s'il est vrai que tu avais entendu dire qu'ils sont impuissants, comment expliquer qu'il ne te soit pas venu à l'esprit qu'il fallait prendre garde? Avais-tu donc oui ou non entendu amatores mulierum esse maximos? Cela même aurait dû te mettre en garde. Car il ne suffit pas d'avoir évité l'attentat à la pudeur, alors que même un seul baiser peut dépraver une femme que tu ne voudrais pour rien au monde voir touchée ou souillée. À moins que uerum ne soit pas une conjonction, mais un nom : elle aurait entendu la première proposition en considérant que c'était faux, sans se douter que la seconde était vraie? De fait, in mentem uenire ne signifie pas seulement reminisci (se souvenir), mais aussi cogitare (réfléchir)»).

19. Valette-Cagnac 1997, p. 136-137, montre bien la fonction d'«émondage » qu'exerce sur le futur texte la lecture publique ou la déclamation. Suivant les réactions de la partie docte du public, l'auteur va corriger et fixer en texte le discours qu'il jugera le plus abouti. Voir Pline, Ep. 5, 3, 10: Atque adeo si cui forte eorum qui interfuerunt curae fuerit eadem illa legere, intelleget me quaedam aut commutasse aut praeterisse, fortasse etiam ex suo iudicio, quamvis ipse nihil dixerit mihi ( « et ainsi, si quelque personne qui était présente a eu la curiosité de lire ce que j'ai déclamé, il comprendra que j'ai fait quelques changements ou suppressions peut-être en suivant son avis, bien qu'il ne m'ait rien dit lui-même »).

20. Voir cependant ci-dessous la question de la gestion possible par l'auteur lui-même de la dimension textuelle de son œuvre, y compris dans la mise en scène de sa propre écriture, voir Ker 2004, p. 227 : «when a writer brings the image of himself writing at night before the eyes of his reader, the text alone is the medium of interaction [...] the resulting performance is programmatic for the reception of each given text ".

21. On ne saurait objecter à cette idée les conseils que Quintilien donne quant au soin à apporter à l'écriture (voir Ker 2004, p. 213 sq.). En effet, écrire, puis raturer, construire chaque phrase et la ciseler au stylet n'implique pas que le but ne soit pas de produire un discours (voir aussi McDonnell 1996, p.470). La recitatio sera pour le poète, l'historien ou le philosophe, le même genre d'épreuve du feu que le forum pour l'orateur. 
22. À ce propos, Valette-Cagnac 1997, p.66, analyse de façon particulièrement éclairante la fonction du lector, esclave ou salarié, dont le rôle est de faire la lecture : «l'existence de ce personnage est [...] significative d'une certaine fonction attribuée à l'écriture et d'une conception de la lecture, où, pour être intelligible, le sens doit être véhiculé, transmis, passer par le détour de l'ouie ». Voir également p. 69 sur la lecture pour soi et le Vorlesen ou lecture pour autrui. Les deux restituent la discursivité, mais dans un cas sans communication réelle, dans l'autre dans la communication entre le lector et l'auditoire.

23. D'ailleurs le verbe scribo en contexte de commentaire entre en corrélation avec des formules $\mathrm{du}$ type legitur, legere debemus, qui relèvent de la perception purement textuelle du texte littéraire, mais dans sa dimension essentiellement ecdotique. Scripsit signifiera par ex. que l'auteur a voulu expressément telle graphie, pour des raisons d'usage ou de convenance personnelle, et legitur, que l'on trouve deux ou plusieurs versions du même texte entre lesquelles le commentateur avisé peut établir une hiérarchie ou qu'il peut simplement proposer comme des leçons alternatives. Sur ces questions et le vocabulaire de l'écriture à Rome, voir la mise au point synthétique de McDonnell 1996, p. 482 sq., et en particulier p.486: "First, there was the production of a draft copy. To indicate this stage, Fronto had manu scripta e\{xem\}pla and his list of famous scholar-copyists. (As a high-class ghostwriter of senatorial speeches Aelius Stilo constituted a special category.) Second, there was a stage of correction, criticism, and revision undertaken first with assistants or close friends, then by a somewhat larger group of friends. Fronto represented this stage with exempla [...] a Tirone emendata. Third, at last, multiple copies were made of the final, polished version by the author's own scribes, or if he was lucky, by a friend's librarii who had a reputation for producing good and accurate copies. "

24. Cela vaut également pour le commentaire qui résulte au départ de la transformation à un degré non mesurable aujourd'hui de l'enseignement oral du maître. Voir Holtz 2000, p. 103.

25. Sur la présence possible de l'auteur lui-même dans cette phase, et l'importance de l'écriture par l'auteur lui-même, voir McDonnell 1996, p. 472 sq.

26. Cette restriction peut paraître saugrenue, mais Donat par ex. considère que la mémoire est le moyen le plus sûr de tous pour conserver quelque chose. Bien que le passage en question n'évoque pas directement la dimension de l'écrit, on a l'impression que, pour le grammairien, le rapport que nous établissons, nous, entre discours et texte (uerba uolant scripta manent) peut s'inverser dans son point de vue : An. 40 : «IN MEMORIA HABEO plus dixit 'in memoria habeo', quam si dixisset 'scio'. nam quae scimus, possumus obliuisci, quae uero memoriae mandamus, numquam amittimus » (« IN MEMORIA HABEO in memoria habeo (j'ai en mémoire) est plus explicite que scio (je sais), car ce que nous savons, nous pouvons l'oublier, mais ce que nous confions à la mémoire, jamais nous ne le perdons »).

27. Sur ces éléments cicéroniens et leur dimension dans le cadre même de la société romaine, voir le très stimulant article de Gurd 2007, où l'auteur analyse le processus social de l'écriture, dans le contexte précis des luttes politiques du milieu du i ${ }^{\mathrm{er}}$ siècle avant notre ère.

28. Sur cette question, voir par ex. Ledentu 2004, p. 359-360.

29. Sur le rôle même des grammatici dans la réalisation de copies, voir McDonnell 1996, p. 477-480, à partir de la discussion d'une lettre de Fronton, Aur. 1, 7, 4.

30. État de cette question chez Valette-Cagnac 1997, p.11-19. L'auteur (p. 29-30) rappelle d'ailleurs fort utilement que l'ordre même des opérations de lecture est le plus souvent à Rome l'inverse de ce qu'il est dans l'éducation moderne : on lit avec les yeux pour apprendre comment ensuite bien déclamer, alors que, dans la perspective moderne, la lecture oralisée est le stade préparatoire à la seule lecture considérée comme experte, la lecture silencieuse. «La lecture silencieuse est donc utilisée 'en parallèle avec' la lecture à haute voix ; à la rigueur comme substitut, jamais comme aboutissement » (p. 32).

31. Voir par ex. Don. Ter., Ph. 55, 2 : SI QVIS QVID REDDIT MAGNA H. E. G. hoc qui interrogatiue pronuntiauerit, parum belle explicat conceptam sententiam ( SI QVIS QVID REDDIT MAGNA HABENDA EST 
GRATIA supposons que quelqu'un prononce cela de façon interrogative, il ne rendra que trop imparfaitement compte de la tournure de l'idée»). Plus clairement lié encore à l'actio, on peut citer cette autre remarque du commentateur dans la même pièce (Ph. 49): VBI INITIABVNT hoc uultuose pronuntiandum (« VBI INITIABVNT il faut prononcer cela avec un air de circonstance, [en l'occurrence ici exaspéré] »).

32. Même dans les recitationes, la règle est en effet de laisser lire le lecteur à son rythme sans interrompre la performance (Valette-Cagnac 1997, p. 132-133), même si l'on sait que certains passages étaient bissés, le public manifestant bruyamment jusqu'à ce que le lecteur (souvent d'ailleurs l'auteur lui-même) relise le passage réclamé (sur les manifestations du public, voir Pline, Ep. 1, 13, 3). On trouve une trace amusante de cette pratique dans la notice qui accompagne dans plusieurs manuscrits l'œuvre du poète Arator. Prévue pour être déclamée en un ou deux jours au printemps 544 à Rome, la séance s'étendit en réalité sur quatre, tant le public réclama de bis. La nature très particulière de ces relectures ne contredit évidemment en rien le fait que le discours demeure fondamentalement linéaire. On voit mal un orateur sur le forum répéter son exorde uniquement pour faire plaisir à l'assistance et, de plus, de telles interruptions en viendraient rapidement, malgré la fierté qu'il pourrait en concevoir, à gêner le lecteur même, car la cohérence du propos pourrait ne plus être perçue. Sur la pratique de la recitatio et le type de textualité qu'elle induit, voir Valette-Cagnac 1997, p. 116-130.

33. Avec le passage au codex, cette manipulation du texte devient encore plus facile. On peut marquer la page que l'on aime, y revenir plusieurs fois, etc. Toutefois, comme le note Valette-Cagnac 1997, p. 37-38, le butinage dans le livre n'est pas une forme de lecture valorisée (cf. Sen., Ep. 5, 45, 1) parce que le discours suit un chemin que le butineur ne parvient plus à suivre correctement dans ses "sauts et gambades ». Au contraire, on peut valoriser une lecture lente et attentive qui revient sur ce qu'elle a lu pour s'en imprégner (Quint., 10, 1, 19), mais on voit bien qu'ici revenir en arrière vise à saisir mieux encore le discours de l'auteur, c'est en quelque sorte ralentir le flux du discours pour mieux le percevoir : Lectio libera est nec ut actionis impetus transcurrit, sed repetere saepius licet, siue dubites siue memoriae penitus adfigere uelis. Repetamus autem et tractemus et, ut cibos mansos ac prope liquefactos demittimus quo facilius digerantur, ita lectio non cruda sed multa iteratione mollita et uelut confecta memoriae imitationique tradatur ( $\mathrm{La}$ lecture est libre, et ne court pas en suivant l'élan de l'action oratoire. On peut revenir à chaque instant sur ses pas, soit que l'on ait un doute, soit que l'on veuille l'inscrire profondément dans sa mémoire. Revenons donc en arrière et manions notre texte, et, comme on mâche longtemps les aliments pour les amollir et ainsi les digérer plus aisément, de même ce que nous lisons ne doit pas être confié à notre mémoire ou à notre imitation tout cru, mais amolli par de nombreuses relectures et comme réduit en bouillie »). Sur l'influence du passage du volumen au codex sur les pratiques de lecture, voir Valette-Cagnac 1997, p. 52-62, en particulier p. 56-57. L'auteur souligne que la « révolution » dans les pratiques de lecture que certains veulent rendre contemporaine de la généralisation du codex a dû en réalité être beaucoup plus lente et que le modèle ancien de discursivité du texte a perduré largement au-delà de la généralisation du codex. De plus, le fait qu'il existait depuis déjà longtemps des formes de cahiers ou de codices avant qu'on se mette à utiliser cet objet comme support préférentiel des livres contribue à relativiser grandement la radicalité de mutation des pratiques de lecture et donc de considération du texte.

34. Sur ce point, voir Tura 2005, p. 276-282.

35. De toute évidence ces deux éléments faisaient défaut aux exemplaires les plus anciens, le texte se présentant en continu, avec peut-être une mention à la fin de chaque acte, et encore. Donat se demande à plusieurs reprises à qui il faut attribuer telle ou telle réplique (signe que les copistes impériaux ou tardifs avaient dû avoir une interprétation différente de l'absence d'indication sur les exemplaires anciens) et précise que déterminer la fin d'une scène ou d'un acte est affaire de spécialiste, ce qui serait absurde si les exemplaires qui circulaient dans son milieu étaient tous d'accord sur la question. Sur l'attribution des répliques, voir Don. Ter., Ad. 287 
par ex. Sur la question de la séparation des actes et des scènes, voir Don. Ter., Hec. Praef. 3, 6, Ad. Praef. 3, 7, et surtout An. Praef. 2, 3.

36. Bien que beaucoup plus tardif, le Térence de Tours (Tours, B.M. 924, vers 1100, visible sur le site http://www.enluminures.culture.fr/documentation/enlumine/fr/) montre clairement que le choix du moment de la pièce qui est représenté ne doit rien au hasard, et combine nécessités de la mise en page du codex et volonté de rendre visibles au lecteur des moments clés de la pièce. Chaque peinture en tête de scène donne une vision de l'espace scénique, mais caractérise également parfaitement les personnages et leurs motivations psychologiques. Ainsi on voit Dave tenter d'échapper à Simon ( $\left.f^{\circ} 2 v^{o}\right)$, la détresse de Pamphile $\left(f^{\circ} 3 v^{\circ}\right)$, etc. On pourrait d'ailleurs faire la même remarque sans doute avec les illustrations célèbres du Virgile du Vatican.

37. Comme le note Valette-Cagnac 1997, p.62, «l'opposition entre codex et volumen [...] correspond manifestement moins à une rupture chronologique qu'à une opposition fonctionnelle, permettant deux façons d'appréhender le livre qui ne cessent d'exister tout au long de l'Antiquité : la lecture auditive et la lecture visuelle ». La première, selon l'auteur, entre dans une logique de «réception» des textes littéraires, la seconde a une fonction plus utilitaire. Le commentaire, comme genre frontière entre les deux, peut à ce sujet particulièrement bien témoigner d'une perception romaine de la littérarité.

38. Voir par ex. la célèbre indication de la branche $\alpha$ du Bellum Gallicum de César, où l'on trouve au livre 2: Flavius Licerius Firminus Lupicinus legi, renvoyant à un personnage par ailleurs bien connu (Ennod., Dict. 8, Ep. 2, 15 et 23, et Ep. 3, 15). On connaît aussi l'activité éditoriale de Turcius Asterius (voir Firenze Biblioteca Medicea Laurenziana 39.1 et Vat. lat. 3225, fo 76), etc. Sur tous ces lecteurs correcteurs qui sont à la source des textes transmis au Moyen Âge, et sur ce qu'ils ont transmis, voir infra. Sur la pratique de l'annotation entre intellectuels latins, voir l'exemple du travail éditorial d'Atticus dans Gurd 2007, p. 52, n. 10. Sur la circulation des livres à Rome, voir par ex. Marshall 1976, p. 253 : «It is true that commercial book production was established in Rome by the early first century B.C. and that by Pliny's day bookstores could be found far afield in the provinces, remote from the central markets of the Argiletum and the Vicus Tuscus. Moreover, a sturdy book might last a hundred years or more with careful handling, and Romans came to know the dusty delights of second hand bookshops. But we hear much complaint about the inaccuracies and deficiencies of commercial copies, which were often carelessly prepared and not properly checked against the original before sale ».

39. Rappelons pour mémoire qu'il est probable que Jérôme lisait encore la totalité de l'œuvre de Tacite, puisque dans son Commentaire à Zaccharie, 3, 14, il parle des trente livres de l'historien.

40. Habinek 2005, p. 83 : «Texts are what we study as philologists, but they are and always have been embedded in networks of practices. Indeed, the fantasy that the world is a text, or that there is nothing outside of text, can be understood as an acute manifestation of what Vico long ago called "the conceit of scholars"? that is, the assumption that what one studies, however well and thoughtfully, explains everything ".

41. Voir Tura 2005, p. 311 et 316.

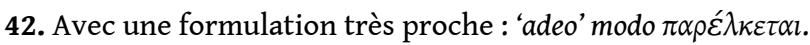

43. Sur la question du statut du grec et des modalités de sa copie, voir Tura 2005, p. 271 (bibliographie aux notes 19-22).

44. Le texte que nous proposons pro arte lecta n'est pas vraiment satisfaisant en l'état, mais il pose au moins la question de la présence de grec dans ce segment, qui nous semble extrêmement sujette à caution. Peut-être faut-il attendre d'avoir une vue complète de la tradition de ce passage précis pour déterminer quel segment latin a pu être corrompu.

45. Gurd 2007, p. 56, montre, en étudiant la controverse autour de la forme Phliuntioi dans le De Republica de Cicéron, que ce type de débat ne concerne pas seulement les grammairiens et existe en réalité dans toute la littérature latine, en tant qu'elle est émanation d'une société cultivée à destination d'une société cultivée. 
46. Habinek 2005, p. 84 : «"to read" in the Roman world (as, indeed in our own, mutatis mutandis), is a practice, entailing a specific, historically constituted set of relationships of body to voice, speaker to listener, male to female, master to slave, owner to object, and so on ».

47. Remarque fondamentale dans sa formulation synthétique chez Gurd 2007, p. 50 : «I will be suggesting that texts embody the interests of social groups through the mechanism of revision. This embodiment may be read as "reflection," though it is in fact the result of a process of collective inscription ». Et note 5: "Literary works usually go through three distinct phases of revision: (1) a phase of "authorial revision," in which the author reads and corrects his own drafts; (2) a phase of "editorial revision," in which the text is submitted to the judgment of other readers, whose advice the author welcomes; and (3) a final phase of "culturalrevision," in which the work, now beyond the control of the author, is appropriated and reformed in adumbrations, imitations, and re-inscriptions ".

48. Gurd 2007, p. 51 : «How does it, as a variable entity, implicate the worlds in which it was copied, reformed, and subjected to different readings and critiques?»

49. Gurd 2007, p. 51 : «We often speak of ancient texts as though they were single objects, stable semiotic systems that, like modern mass-produced books, remain largely unchanged in form. This was manifestly not the case in antiquity, as generations of textual critics have known. Ancient texts are variable systems (or systems of variants), and formal and verbal divergence is the norm, legible in the archive of practically every work. A maximal social interpretation of ancient writing would have to posit not only that texts are engaged in one context, but also that their verbal fluctuations implicate multiple contexts ».

50. « Mise en communication de deux sources disparates», selon Tura 2005, p. 269.

51. Sur ce point et la suite, voir Holtz 1984, et Holtz 2000, p. 104, pour le passage de notes venues d'hypomnèmata dans un commentaire de type marginal. Sur la forme des hypomnèmata grecs, voir Maehler 2000, en particulier p. 34-35: «Il est [...] évident qu'il aurait été tout à fait impossible de transférer le contenu entier d'un hypomnèma antique de cette façon (en notes marginales). Cela n'était possible que par un processus d'abréviation drastique effectué en plusieurs étapes [...] La première étape d'abréviation a probablement été la transcription des textes des rouleaux aux codices [...] Il me semble très probable que cette transcription a été une bonne occasion pour les copistes de se débarrasser d'un tas de matériaux qui n'étaient plus d'actualité, vu que la production de livres était de plus en plus déterminée par les usages de l'école ».

52. Ce modèle n'est pas exclusif, en effet un commentaire comme celui d'Asconius à Cicéron a été pensé pour être séparé de la diorthosis, puisqu'il comprend des indications explicites sur la localisation dans le volumen des passages que le commentateur retient. Voir par ex. dans le commentaire de l'In Pisonem la mention circa vers. LXXX ("vers la ligne 80 »). De même, tout porte à croire que le commentaire virgilien de Tiberius Donat a été conçu comme un texte séparé, un livre sur le livre en quelque sorte. Nous parlons ici de ce qui constitue le commentaire dit grammatical (Donat, Lactantius, Servius, Porphyrion, Pseudo-Acron, et d'autres).

53. Maehler 2000, p.35: «il semble que [...] les besoins de l'enseignement scolaire déterminai[en]t non seulement le choix des textes considérés comme essentiels, choix qui devenait progressivement de plus en plus limité, mais aussi la perspective des usagers ou des 'consommateurs' de livres - d'où, par exemple, le nombre croissant de paraphrases et d'explications mythologiques aux dépens des observations de caractère stylistique ». On observerait un exemple de cette mutation dans le glissement du commentaire de Donat à Térence à celui d'Eugraphius, probablement postérieur, et qui se caractérise par un accent mis davantage sur la paraphrase psychologique des situations que sur le commentaire littéraire, rhétorique et grammatical. Sur le glissement du commentaire à lemmes vers le commentaire marginal et vice versa, voir Holtz 2000, p. 104-105. Sur les traces de ces glissements dans la mise en page ellemême, voir en particulier p. 105.

54. Don. Ter., Hec. 2. 
55. Remarque très importante à ce sujet dans Gurd 2007, p. 63 : «A speech is effective, this line of reasoning implies, in part because the speaker's overall habitus is imbued with the expectations of his community. But Cicero goes a step further: a work must not only embody the languages of its society, it must also call for further involvement and correction. By means of the former, a text is the charter of one community; by means of the latter, it invites the formation of a plural community over time. There is, in other words, an element in Cicero's thinking of the historical community at Rome that is structured by the necessity of a seeming or functional incompletion: a work will only draw a world around it if it is worthy of being formally negotiated, that is, if it seems both strong and incomplete ". Cette idée, à mon sens, éclaire très largement le travail des commentateurs anciens, et la permanente négociation que le lecteur romain mène avec sa propre tradition.

56. Pour ne prendre que quelques exemples bien connus et latins, citons la Rhétorique à Herennius, L'Etna ou l'Octavie.

57. En réalité, l'auteur paraît souvent moins important que le texte lui-même, au moins dans un premier temps. Un poème comme le Panégyrique de Messala est arrivé on ne sait trop comment dans l'œuvre de Tibulle, mais sans doute parce qu'il émane du même cercle que l'authentique Tibulle, Lygdamus et Sulpicia, ou que le même copiste les a rassemblés pour lui-même ou un commanditaire à un certain moment. Ensuite, il est tout aussi intéressant de noter le glissement, sur lequel nous reviendrons plus bas, qui conduit ce poème du corpus Tibullianum à pouvoir être pris pour du Tibulle, comme la Rhétorique à Herennius a pu être prise pour du Cicéron.

58. voir, avec des nuances sur la façon dont est envisagée la notion même de littérarité comme liée à la " grande » littérature, Tura 2005, p. 266 : «Sans diminuer [leur] caractère de spécificité, on peut observer que le degré de littérarité propre des commentaires n'est pas toujours si ténu [...] Le fait qu'il existe un grand nombre d'écrits qui, sans nullement être des commentaires ni en partager le caractère auxiliaire par rapport à d'autres textes, sont néanmoins affectés par un degré presque négligeable de littérarité et, par conséquent, eux aussi soumis à des transmissions qui les métamorphosent [...] L'opposition entre 'commentaries' et 'literary texts' n'a pas plus de sens que de vouloir opposer le jaune au vert, deux termes distincts, non pas contraires. De plus, on peut constater que, s'il ne s'agit pas de textes classiques ou patristiques, la littérarité ne garantit guère que les copistes ne varient pas en toute désinvolture ». On peut remplacer ici avantageusement la notion de désinvolture par celle, sans doute plus positive, de vision de l'œuvre ou du texte copié.

59. Sur les phénomènes induits par cette pratique, voir Tura 2005, p. 310-311.

60. Holtz 2000, p. 103.

61. Sur ce phénomène, voir en particulier Gurd 2007, p. 51 : «First, a text is any single version: if it has been revised by more than one hand it embodies the interests of more than one person. Second, we understand a text to be the complete system of variants, that is, a set of versions united by a certain identity of content. Because it maintains its identity even while changing its details, a text can link a variety of different points of view and establish continuity across a range of positions. In the first sense, a text is the embodiment of the interests of a specific group: this explains its apparent "reflection" of a social milieu. In the second, it is a means of forging continuity across change, actively building a space where differences can be performed, shared, compared, and negotiated. In the light of the broader system of variance that constitutes "text" in the second sense, any single version may be seen as the fragment of a larger whole, valuable not only for its ability to reflect a specific milieu but also for its invitation to later readers to take it up and inform it with their own expectations. In this sense, individual versions capable of fostering broader communities may be described as incomplete or corrigenda ».

62. Tura 2005, p. 305, distingue de façon particulièrement commode " gloses de confection » et « marginalia de lecture ». 
63. Le phénomène est particulièrement patent dans les Scholies de Berne à Virgile. On trouve trace d'un état du texte où les annotations pouvaient être rapportées à leur auteur originel, mais ces attributions auctoriales sont rares et finalement le texte a pu, malgré ses variantes d'un exemplaire à l'autre, arriver à une certaine forme de stabilité où il entre dans une tradition de littérarité. Sur tous ces points, voir, même si ses conclusions doivent être reprises avec précautions, Funaioli 1930.

64. On trouve d'intéressants phénomènes de ce genre chez Prudence par ex. ou chez Sedulius, mais aussi dans une moindre mesure peut-on peut-être en déceler chez Properce, voire dans certaines variantes cicéroniennes. Voir par ex. le cas parfaitement attesté cité à la note 45 . Sur ces « traces de lecture », voir Tura 2005, p. 317-329 et aussi p. 355-363.

65. Holtz 2000, p. 102, souligne clairement que la première forme du commentaire antique était celle d'un mémoire, présenté séparément du texte commenté, à la mode de ce qui se trouve dans nos manuscrits de Donat. La création des lemmes visait alors à relier clairement le mémoire de commentaire au texte lui-même. Ainsi on pouvait lire avec ou sans commentaire, dans une optique d'étude ou de plaisir. On pourrait alors croire que c'est cette tradition qui aboutit directement à nos manuscrits de commentaires, mais il n'en est rien. La forme sous laquelle nos copistes ou leurs archétypes ont trouvé les commentaires est bien celle de gloses inscrites dans le texte lui-même. Comme le montre Holtz, il existait du reste dès l'Antiquité des commentaires de ce type dans lesquels ont pu atterrir également des éléments démembrés de commentateurs existant par ailleurs sous des formes continues. Sur l'histoire complexe de ce processus, voir Holtz 2000, p. $107 \mathrm{sq}$. avec, p. 108, une très importante analyse des conséquences sur le commentaire lui-même des divers changements de mise en page, en comparant Bern BB 172/ Paris BNF 7929 et Bern BB 167. Voir aussi Tura 2005, p. 263.

66. Holtz 2000, p. 110.

67. Sur la question des marginalia, voir Tura 2005.

68. Holtz 2000, p. 104, sépare clairement ce qui relevait des notes marginales et interlinéaires de ce qui relevait de l'explication continue. On voit bien que dans nos manuscrits actuels, tant glosés qu'indépendants, les éléments ont été largement mélangés.

69. On pourrait ici évoquer le cas particulièrement épineux des rapports qu'entretient avec les Scholies de Berne le commentaire de Philargyrius, cité plus haut. On trouve des éléments communs, parfois des scholies entières, dans l'une ou l'autre version du commentaire de Philargyrius et les scholies, mais ni l'état originel du commentaire, ni les mécanismes qui ont conduit là où on la lit telle ou telle bribe.

70. Holtz 2000, p. 101 : «les mises en page médiévales complexes étaient en fait le point d'aboutissement d'une longue évolution mettant en cause indissociablement à la fois la vie intellectuelle et la technique du livre».

71. Sur le traitement de ces contraintes, voir Tura 2005, p. 291-299.

72. Tura 2005, p. 292-294, avec la remarque fondamentale p. 294 sur le moment où la diorthose n'est plus présente: «cela peut entraîner de lourdes conséquences pour la tradition, car des citations de cette sorte peuvent consentir une reconstitution du texte de l'œuvre commentée de la part de quelqu'un qui ne dispose pas d'un témoin direct pour la copie ».

73. Sur ce phénomène, voir Tura 2005, p. 268.

74. Compte tenu évidemment du fait que, dans la plupart des cas, il s'agit probablement de textes retravaillés pour la publication (voir supra). Il n'en demeure pas moins que leur littérarité propre se définit par ce caractère oral qui caractérise la prédication et aussi, assez régulièrement, par le choix d'un niveau de langue qui ne soit pas accessible seulement aux savants et aux auditeurs raffinés, mais qui soit intelligible par l'ensemble de la plebs Romana.

75. Holtz 2000, p. 106, note à ce sujet : «Jérôme se trouve au carrefour de plusieurs traditions [...] au point de jonction entre les traditions de l'école romaine et celles de l'exégèse chrétienne qu'il contribue à instaurer en Occident. Et c'est lui-même qui, emporté par sa fougue de polémiste, 
proclame, et cela au moins à sept reprises dans le cours de son œuvre, que quel que soit le texte commenté, profane ou chrétien, la méthode du commentaire est la même, car le commentaire a ses lois, leges commentariorum. »

76. Sur l'auteur et son œuvre, voir le classique O'Donnell 1979, en particulier le chapitre v.

77. À titre de comparaison on peut citer la glose ordinaire qui n'a jamais connu ce type de mutations, mais a été d'emblée conçue comme une annotation marginale. Voir Holtz 2000, p. 112-114.

78. Je rejoins ici pleinement l'observation de Gurd 2007, p.64, pour qui cette dimension est constitutive d'une grande partie de la conception romaine de la littérature : «Collective revision, in other words, is not only a means of fostering sociality; it is the very medium of that sociality, since by correcting the work of others we join with them in a trans-temporal rhetorical community ». On trouverait trace de cette idée tout au long de l'Antiquité, par ex., à sa toute fin, dans les échanges critiques de Sidoine Apollinaire avec Fauste de Riez, ou dans les nombreuses discussions littéraires des correspondances tardives.

79. Sur tous ces points, voir Robinson 2004.

80. L'apparat critique rend évidemment compte d'une forme de diachronie, mais il a avant tout pour but de justifier le texte édité et non d'établir le sens possible des variantes rejetées et leurs conséquences sur l'interprétation même du texte. Dans de nombreuses éditions commentées, depuis la Renaissance, on a pris l'habitude d'insérer les références aux commentateurs antérieurs en usant d'ailleurs d'une méthode qui ressemble à ce qu'on trouve par ex. dans certaines des Scholies de Berne aux Bucoliques. Toutefois, dans tous les cas, la forme même de l'objet livresque oblige à ne fournir que des extraits, lemmes pour l'apparat, citations pour le commentaire. Le choix de ce qui est visible revient donc ultimement au seul éditeur et l'interaction immédiate possible par l'utilisateur est minime.

81. Par ex. dans le cas d'un texte oublié et récemment redécouvert comme le fragment du livre 2 de Rutilius Namatianus découvert en 1973, voire même l'ensemble du poème qui demeura inconnu du Moyen Âge, ne fut découvert qu'à la toute fin $d u x^{e}$ siècle et ne fut connu que par un seul manuscrit, aujourd'hui disparu.

82. Les deux meilleurs exemples de cette littérarité complexe multiforme et largement diachronique sont évidemment Homère et Virgile et la masse des variantes, commentaires, gloses, etc., que l'Antiquité même nous a laissés, pour ne rien dire du Moyen Âge et de l'époque moderne. Savoir exactement comment Dante lisait Virgile, autrement dit quelle était la littérarité que l'on donnait à Virgile en son temps, n'est qu'un exemple parmi d'autres de l'importance de la reconstruction des Virgile, et non du seul Virgile, poète augustéen.

83. Voir par ex. http://am-dk.net/fasnl/texts/viewer.php?id=hsk.

84. Sur l'importance de l'analyse du support dans le cas des textes présentant des gloses, voir Tura 2005, p. 264, qui a cette remarque fondamentale à nos yeux : " Tout manuscrit est, dans sa confection, l'espace ambiant de nombreux choix de la part du copiste ou de celui qui, en lui confiant la transcription, lui en prescrit les modalités. De ces choix relève la répartition en divers rangs des textes que l'on décide de juxtaposer. L'un des principaux intérêts que l'étude des manuscrits peut offrir dépend justement du fait que, loin de reproduire mécaniquement des relations préétablies entre les différents textes (de primauté, de subordination, etc.), chacun est la source d'une nouvelle organisation, de sorte qu'on peut dire que cette organisation est, pour tout manuscrit, entièrement endogène. Bien souvent, d'ailleurs, un manuscrit ne cesse, même longtemps après sa confection, d'être le lieu d'émergence d'organisations tout à fait nouvelles entre différents textes, que ce soit en subissant des altérations structurales, ou simplement en étant enrichi de quelques écrits dans ses marges. "

85. Ce qui, rappelons-le, n'est pas le cas de Donat; les deux seuls manuscrits antérieurs au $\mathrm{XV}^{\mathrm{e}}$ siècle étant lacunaires, il ne reste qu'une quarantaine de manuscrits de ce siècle, certains étant d'ailleurs copiés sur des éditions imprimées. Sur tout cela, voir Bureau, Nicolas (éds) 2010. 
86. Cesena, Biblioteca Malatestiana XXII $11 \mathrm{~V}, M$ dans notre édition.

87. L'imprimerie justement réduit ce besoin de comparer les exemplaires, mais toute personne qui a eu en mains des éditions anciennes sait bien que le travail de normalisation des éditions imprimées a été long et parfois difficile, et qu'il n'y a finalement pas si longtemps que l'on peut atteindre à une certaine standardisation des textes, au moins modernes. On ignore évidemment comment réagiraient face à des Montaigne «en français moderne » des «éditeurs » qui seraient par rapport à nous dans le même écart temporel que nous le sommes vis-à-vis de Térence ou de Virgile.

88. On peut remonter plus haut avec les papyri, mais ils sont très souvent extrêmement lacunaires et ne transmettent finalement qu'une part réduite du peu que nous avons déjà conservé de la littérature gréco-romaine.

\section{RÉSUMÉS}

La coexistence actuelle du livre et des objets numériques, parce qu'elle interroge le statut du livre et donc de la littérarité qu'il est censé contenir, invite, pour sortir d'une pure logique d'opposition, à s'interroger à nouveau sur ce qui donne, depuis les Anciens, son caractère littéraire à un texte. L'article envisage cette histoire sous l'angle de la prise en compte des conditions matérielles de la transmission du discours devenu texte en montrant que toute édition comporte en réalité sa propre réflexion sur la littérarité des textes concernés, et qu'ainsi la littérarité d'un texte ancien peut aussi se penser en termes de succession de littérarités.

\section{INDEX}

Mots-clés : auctorialité, commentaire antique, ecdotique, gloses, grammairiens latins, lecture, linéarité, littérarité, note alexandrine, numérique, oralité, philologie, pratique éditoriale antique et médiévale, pratique oratoire, support textuel, tradition interprétative, variante manuscrite Keywords : authorship, ancient commentary, ecdotic, glosses, Latin grammarians, reading, linearity, literariness, Alexandrian notes, digital editions, oral, philology, ancient and medieval editorial practice, oratorical practice, textual support, interpretative tradition, handwritten variant

nomsmotscles Ammien Marcellin, Augustin, Ausone, Boèce, Cassiodore, César, Cicéron, Claudien, Donat, Ennode de Pavie, Fatosus, Fauste de Riez, Gallus, Guillaume de Conches, Jérôme, Lactantius Placidius, Lygdamus, Martianus Capella, Palladius, Pélagonius, Philargyrius, Pline le Jeune, Porphyrion, Prudence, Pseudo-Acron, Quintilien, Rémi d'Auxerre, Rutilius Namatianus, Sénèque, Servius, Sidoine Apollinaire, Sulpicia, Tacite, Térence, Tibulle, Végèce, Virgile

\section{AUTEURS}

\section{BRUNO BUREAU}

Faculté des Lettres et Civilisations, Université Jean-Moulin Lyon 3 